\title{
Hijacking a key chromatin modulator creates epigenetic vulnerability for MYC-driven cancer
}

\author{
Zhenhua Yang, ${ }^{1}$ Kushani Shah, ${ }^{1}$ Theodore Busby, ${ }^{1}$ Keith Giles, ${ }^{1}$ Alireza Khodadadi-Jamayran, ${ }^{1}$ Wei Li, ${ }^{1,2}$ and Hao Jiang ${ }^{1,2}$ \\ 'Department of Biochemistry and Molecular Cenetics, UAB Stem Cell Institute, University of Alabama at Birmingham School of Medicine, Birmingham, Alabama, USA. ²Department of Biochemistry and \\ Molecular Genetics, University of Virginia School of Medicine, Charlottesville, Virginia, USA.
}

\begin{abstract}
While the genomic binding of MYC protein correlates with active epigenetic marks on chromatin, it remains largely unclear how major epigenetic mechanisms functionally impact the tumorigenic potential of MYC. Here, we show that, compared with the catalytic subunits, the core subunits, including DPY30, of the major H3K4 methyltransferase complexes were frequently amplified in human cancers and selectively upregulated in Burkitt lymphoma. We show that DPY30 promoted the expression of endogenous MYC and was also functionally important for efficient binding of MYC to its genomic targets by regulating chromatin accessibility. Dpy30 heterozygosity did not affect normal animal physiology including lifespan, but significantly suppressed Myc-driven lymphomagenesis, as cells failed to combat oncogene-triggered apoptosis as a result of insufficient epigenetic modulation and expression of a subset of antiapoptotic genes. Dpy30 reduction also greatly impeded MYC-dependent cellular transformation, without affecting normal cell growth. These results suggest that MYC hijacks a major epigenetic pathway - H3K4 methylation - to facilitate its molecular activity in target binding and to coordinate its oncogenic program for efficient tumorigenesis, meanwhile creating "epigenetic vulnerability." DPY30 and the H3К4 methylation pathway are thus potential epigenetic targets for treating certain MYC-driven cancers.
\end{abstract}

\section{Introduction}

C-MYC (hereafter referred to as MYC) is an important oncogene that is hyperactivated and a central driving force in a wide spectrum of human cancers (1). MYC can be activated through gene amplification or translocation, the latter of which is probably best exemplified by human Burkitt lymphoma (BL) featuring $M Y C / I g$ chromosomal translocations $(2,3)$. E $\mu$-Myc-transgenic mice develop aggressive B cell lymphomas as a result of $M y c$ transgene activation under the control of the $\operatorname{Ig} E \mu$ enhancer (4) and have been a highly valuable model for studies of human BL and for understanding mechanisms regulating MYC-driven tumorigenesis $(5,6)$.

MYC drives tumorigenesis mainly by acting as a transcription factor that binds to numerous genomic sites and regulates the expression of a large number of target genes (7). While MYC has been proposed to be a universal amplifier of all activated genes in the genome $(8,9)$, it is also argued that the selective regulation of transcription of different targets by MYC underlies the role of MYC in tumorigenesis $(7,10,11)$. In either model, efficient binding of MYC to its genomic targets is invariably crucial to its oncogenic activity. While it has been long known that MYC recognizes the E-box element in the genome, a better correlation was shown between MYC-binding sites and epigenetic marks associated with active transcription in the genome, such as histone H3 K4 and -K79 methylation and H3 acetylation $(12,13)$. However, out-

Authorship note: ZY and KS contributed equally to this work. Conflict of interest: The authors have declared that no conflict of interest exists. Submitted: August 24, 2017; Accepted: May 29, 2018.

Reference information: J Clin Invest. 2018;128(8):3605-3618.

https://doi.org/10.1172/JCI97072. standing questions remain regarding (a) the causal relationship between MYC binding and occupancy of the epigenetic marks, (b) whether MYC and the relevant epigenetic modulators regulate each other, and (c) what the functional impact and significance of such regulation might be.

Histone H3K4 methylation is not only one of the most prominent epigenetic marks associated with active or poised transcription (14), but also functionally regulates chromatin transcription $(15,16)$. As the major H3K4 methylation enzymes in mammals, the SET1-MLL complexes comprise SET1A or -1B or MLL1, -2, -3 , or -4 as the catalytic subunit, and WDR5, RbBP5, ASH2L, and DPY30 as the integral common core subunits necessary for complete methylation activity (see diagram in Figure 1A) (17). Human $M L L 1$ is a common target of chromosomal translocations in acute leukemias, and genetic lesions and/or altered expression of other subunits are extensively associated with a variety of human cancers (18-26). Compared with the large number of correlative studies, functional evidence of these subunits in tumorigenesis is rather limited, and the role of $\mathrm{H} 3 \mathrm{~K} 4$ methylation activity in tumorigenesis remains elusive.

We have previously established a direct role of DPY30 in facilitating genome-wide $\mathrm{H} 3 \mathrm{~K} 4$ methylation, especially $\mathrm{H} 3 \mathrm{~K} 4$ trimethylation (H3K4me3) (27), which allows us to investigate the functional roles of efficient H3K4 methylation in physiological and pathological processes by genetically manipulating DPY30. Further studies by us and others have identified important roles of DPY30 in regulating fundamental cellular processes including cell growth $(28,29)$, differentiation $(27,28)$, and senescence $(29)$, as well as stem cell fate determination $(27,30,31)$. While we have previously shown that DPY30 directly regulates the expression of 
A

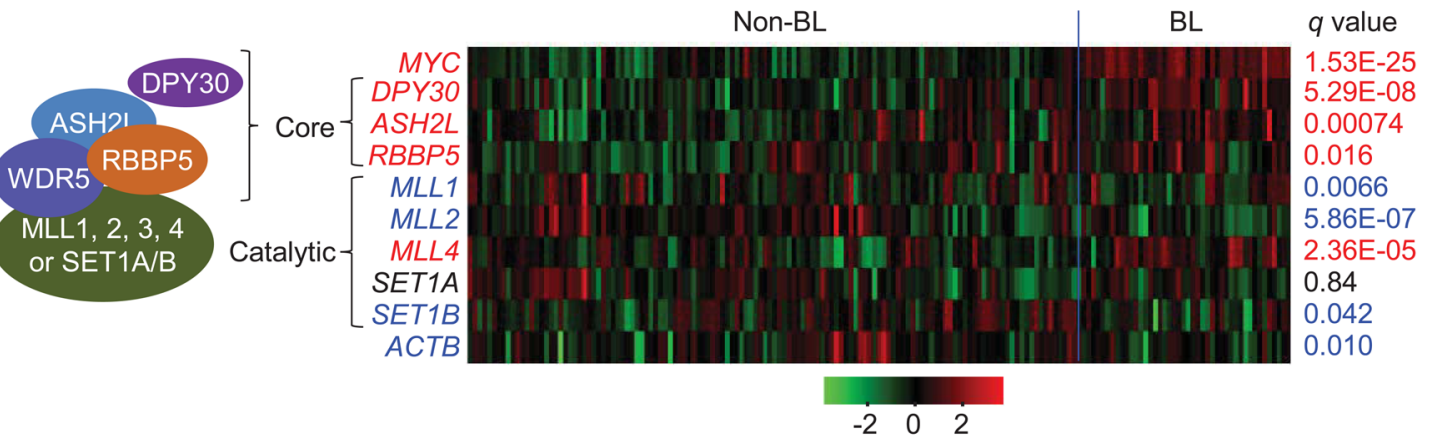

Column Z score
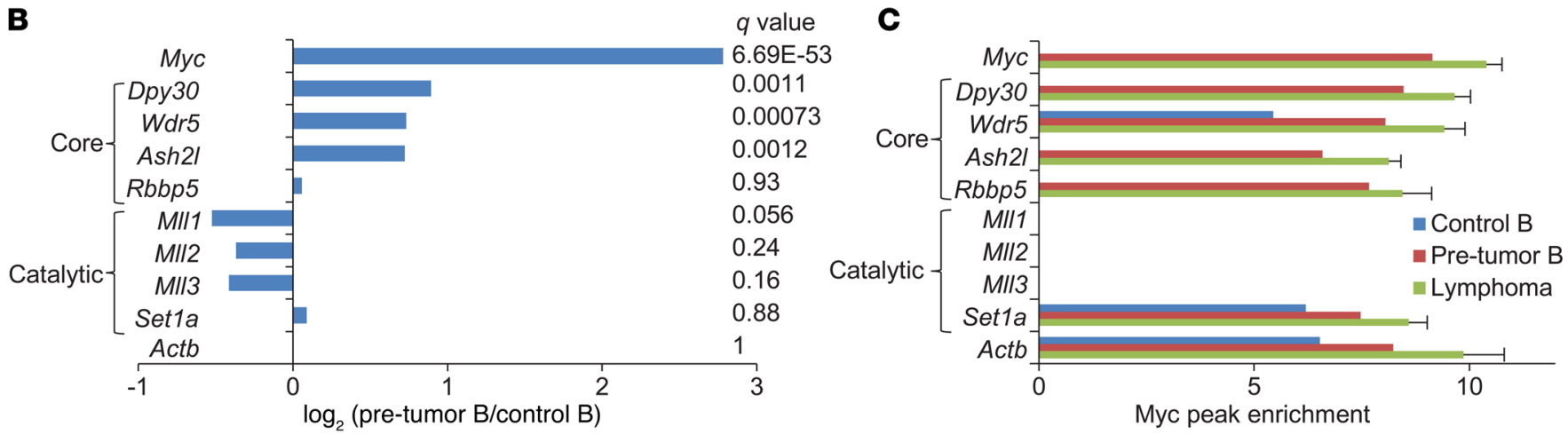

Figure 1. MYC directly promotes the expression of core subunits in SET1-MLL complexes. (A) Expression of SET1-MLL complex subunits in 44 primary human BL samples versus 129 non-BL samples from GEO GSE4475. Each column represents a sample, and each row represents the indicated gene. Genes in red indicate increased expression in BL, and genes in blue indicate decreased expression in BL. $q$ values (FDRs) of the expression changes between non$\mathrm{BL}$ and $\mathrm{BL}$ samples are listed on the right. The color key at the bottom reflects the expression level. (B and $\mathbf{C}$ ) Analyses of data from $E \mu-M y c$ mice from GEO GSE51011. (B) Expression changes of SET1-MLL complex subunits from nontransgenic control B cells to pretumor B cells in young E $\mu$-Myc mice. $q$ values (FDRs) of the expression changes between 4 pretumor and 4 control samples are listed on the right. (C) Myc binding to genes encoding the SET1-MLLcomplex subunits in the indicated samples, shown by Myc ChIP signal peak enrichment values (mean \pm SD) at the promoters of the indicated genes from 1 control, 1 pretumor, and 3 tumor samples.

the endogenous MYC gene and is important for the growth of several MLL-rearranged leukemia cell lines in culture (28), a role for DPY30 in cancer has not been further demonstrated. In this work, we set out to address a functional role of DPY30 in regulating the molecular activity of MYC in tumorigenesis.

\section{Results}

The SET1-MLL complex core subunits including DPY3O are upregulated in BL. To systematically examine the roles of the different SET1MLL complex subunits in human cancers, we started by analyzing their genetic alterations using the cBio Cancer Genomics Portal (http://www.cbioportal.org/) (32). Surprisingly, our analyses revealed that, while most of the catalytic subunits were frequently mutated, the core subunits were often amplified across a wide variety of human cancers (Supplemental Figure 1; supplemental material available online with this article; https://doi.org/10.1172/ JCI97072DS1). Such alterations suggest tumor-suppressive roles of the catalytic subunits, but possibly tumor-promoting roles of the core subunits of the SET1-MLL complexes.

As differential functions often require differential regulation, we sought to identify the regulatory mechanisms of these subunits related to oncogenesis. We have previously shown that high levels of MYC directly promote the expression of several subunits of the
Set1-Mll complexes, especially Wdr5 and Dpy30, in mouse embryonic fibroblasts (MEFs) (30). To assess the impact of MYC on the expression of these methylation modulators in a more clinically relevant setting, we queried expression data sets of primary human BL samples with $M Y C / I g$ translocations versus those of non-BL subtypes (33). We found that the expression of the core (including DPY30, ASH2L, and RbBP5), but not the most catalytic, subunits of SET1-MLL complex was significantly upregulated in BL samples versus non-BL subtypes (Figure 1A). Using data sets from the $E \mu-M y c$ lymphomagenesis mouse model (10), we again found that expression of the core, but not the catalytic, subunits was upregulated in the $E \mu-M y c$ pretumor B cells compared with that in control B cells (Figure 1B and Supplemental Figure 2). Moreover, Myc bound to genes encoding the core, but not most catalytic, subunits in $E \mu-M y c$ pretumor B cells and lymphomas (Figure $1 \mathrm{C}$ and Supplemental Figure 2). These results indicate that expression of the core subunits of SET1-MLL complexes is directly and selectively promoted by MYC, indicating their potential role in the function of MYC in tumorigenesis. Such differential regulation befits the divergent alterations of these subunits in human cancer.

Further analyses of a wide variety of cancer types in the cBio Cancer Genomics Portal showed that nearly $50 \%$ of studies (among studies with sufficient sample numbers with available 
A

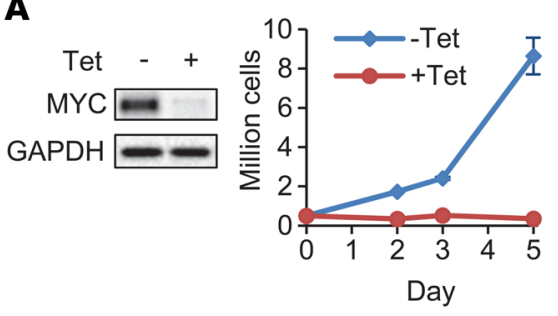

D

No Tet

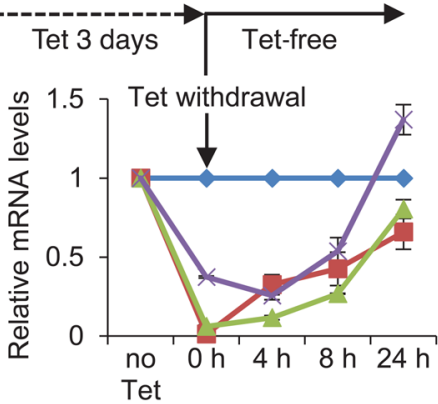

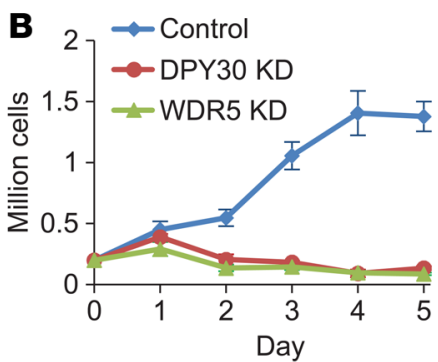

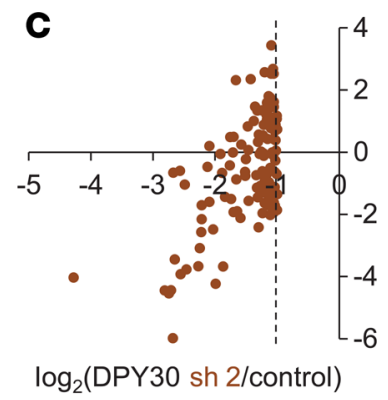

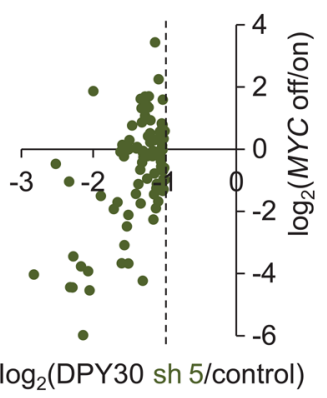
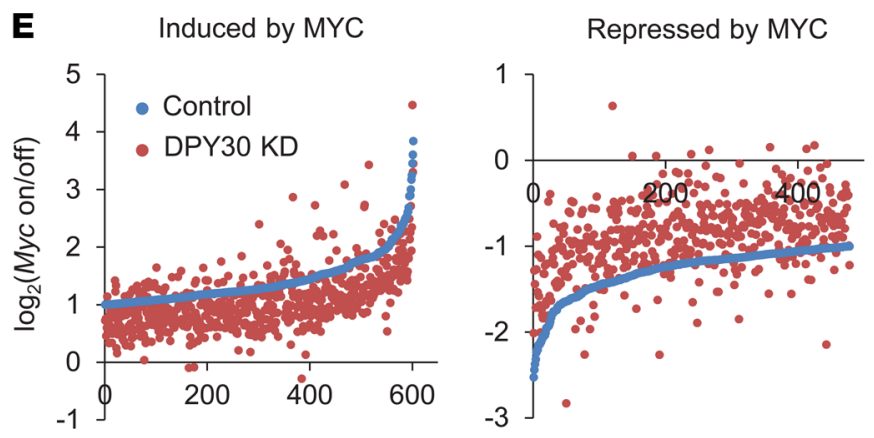

Figure 2. Dpy30 regulates the expression of MYC targets. (A) Immunoblot showing MYC silencing by tetracycline. Graph shows growth curves of P493-6 cells in the presence or absence of tetracycline. Cell numbers and the mean \pm SD from 2 independent platings are plotted. (B) Growth of P493-6 cells following DPY30 or WDR5 KD. Cell numbers were counted, and the mean \pm SD from 8 independent measurements are plotted from a representative assay of more than 8 (for DPY30 KD) or more than 4 (for WDR5 KD) independent KD and growth assays. (C) Codependence of many genes on DPY30 and MYC. P493-6 cells were either depleted of DPY30 by 1 of 2 different shRNAs (sh 2 and sh 5 ) or cultured with tetracycline for 3 days to turn off MYC, and the expression changes were analyzed by microarray. For genes downregulated over 2-fold by DPY30 KD using each shRNA, logarithmic fold changes of gene expression upon DPY30 KD are plotted against their expression changes after turning MYC off. Each dot represents a gene. (D) P493-6 cells were cultured in the presence of tetracycline for 3 days, followed by tetracycline withdrawal and culture in tetracycline-free medium. Relative mRNA levels of the indicated genes at different time points were determined by qPCR and normalized to ACTB. Data indicate the mean \pm SD from duplicate measurements and are representative of 2 independent assays. (E) Control (scramble shRNA) and DPY30-KD P493-6 cells were treated according to the scheme in D, and cells at 0 and 4 hours after tetracycline withdrawal were used for microarray analyses. Logarithmic fold changes from 0 to 4 hours (from MYC off to 4 hours of MYC reactivation) are plotted for the genes upregulated by more than 2-fold in control cells (left) and for genes downregulated by more than 2 -fold in control cells (right). Each dot represents a gene.

data) found notable elevation of DPY3O expression in tumors with increased copy numbers of at least 1 of the $3 M Y C$ genes (C-MYC, N-MYC, and L-MYC) (Supplemental Figure 3A). Therefore, the association between $M Y C$ and DPY3O overexpression is not tumor-type specific. This is consistent with our conclusion that MYC binds to the DPY3O gene and directly promotes its expression. DPY3O overexpression and MYC amplification/overexpression do not necessarily overlap in patients' tumor samples (Supplemental Figure 3B), suggesting a complex regulation of DPY3O expression by $M Y C$ and other mechanisms in vivo.

$D P Y 30$ regulates the expression of MYC and MYC target genes. To study a functional role of the SET1-MLL complex core subunits in MYC-driven tumorigenesis, we started with Raji, a BL cell line, and Jurkat, an acute $\mathrm{T}$ lymphoblastic leukemia cell line that expresses (34) and is dependent (35) on a high level of MYC. We found that the growth of both cells was significantly inhibited by DPY30 knockdown (KD) (Supplemental Figure 4A). Moreover, the expression of $M Y C$ and $M A X$, which encodes an important partner of MYC (36), was significantly reduced upon DPY30 KD (Supplemental Figure 4, B and C). This was consistent with our previous findings that DPY30 directly regulates the endogenous MYC expression in human MLL1-rearranged leukemia cells (28).
To facilitate further functional studies, we used P493-6 cells, a model of human BL cells that express a tetracycline-repressible MYC transgene but a negligible level of endogenous MYC (37). We found that, as occurred when MYC was turned off (Figure 2A), DPY30 or WDR5 KD abolished the growth of these cells (Figure 2B). DPY30 KD greatly reduced the proliferation of P493-6 cells, with little effect on apoptosis (Supplemental Figure 5, A-D). Consistent with these cellular phenotypes, our microarray results (Supplemental Figure 5E) showed that DPY30 KD in P493-6 cells downregulated a subset of genes involved in cell-cycle progression, such as CCNA2, CCND2, MCM1O, CDCA7, and CDCA5, but not most of the other prosurvival genes (except for BIRC5).

Further analysis of the microarray results (Figure $2 \mathrm{C}$ and Supplemental Table 1) revealed a correlation between the effects of DPY30 $\mathrm{KD}$ and MYC silencing on gene expression. Most genes that were markedly downregulated by DPY30 KD were also downregulated by MYC silencing (Figure 2C), supporting the idea of a coregulation of a large subset of genes by DPY30 and MYC. We next assessed the impact of DPY30 loss on MYC-mediated global gene reprogramming. We turned off MYC expression by tetracycline and then turned it back on by washing tetracycline away (Figure 2D). While turning MYC off downregulated the expression of WDR5 and DPY3O, 
A

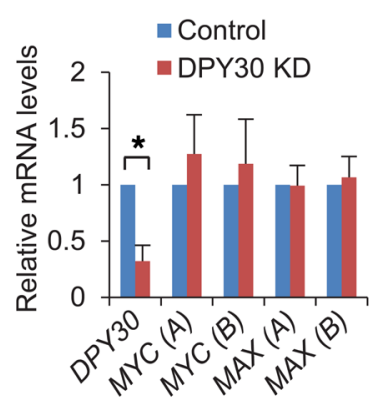

B

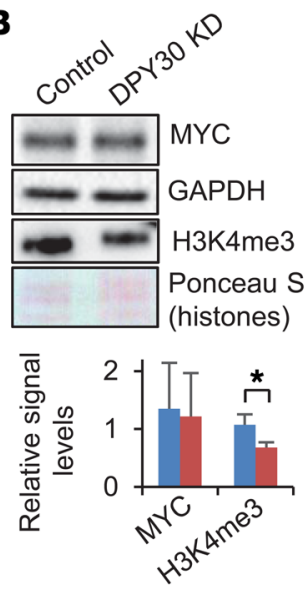

C

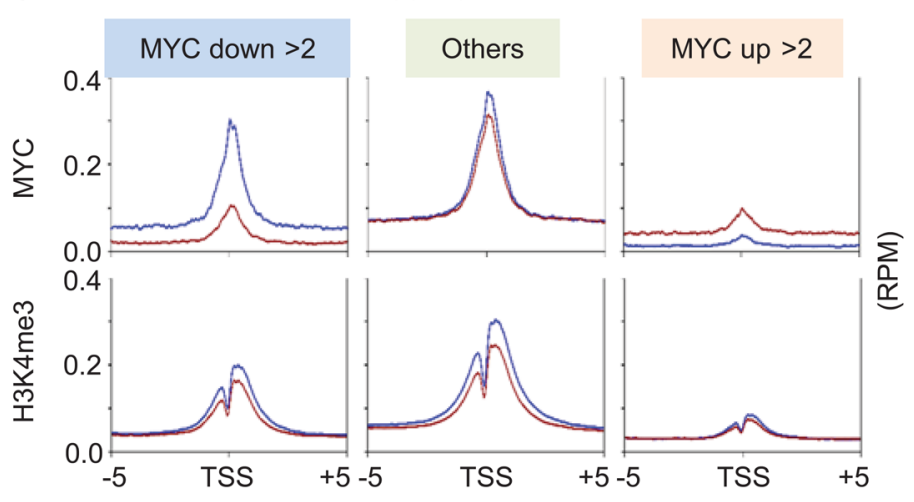

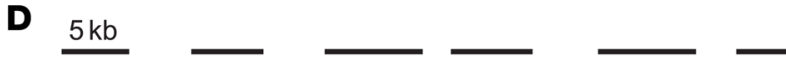

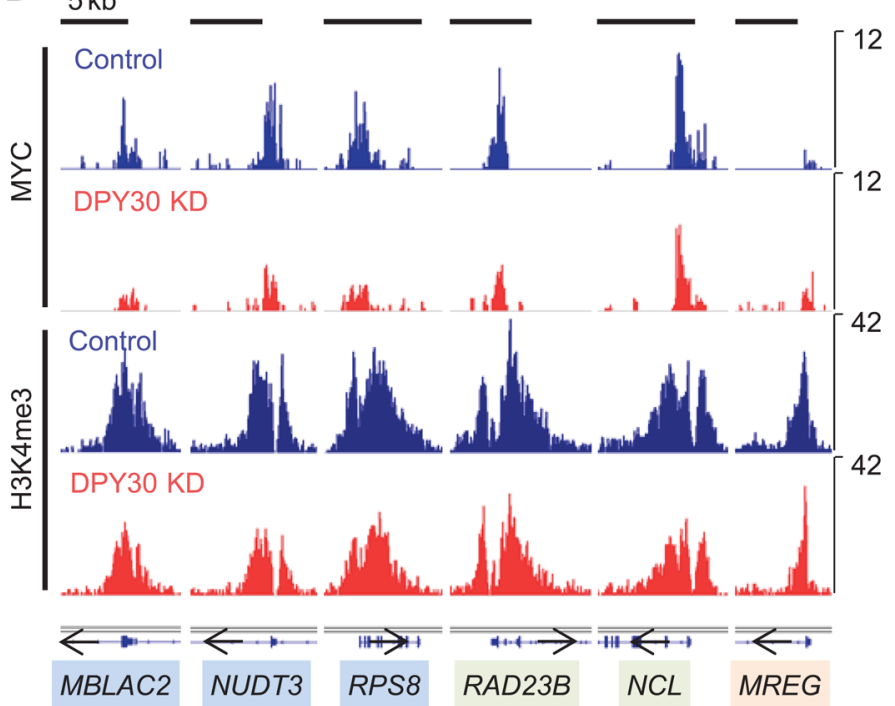

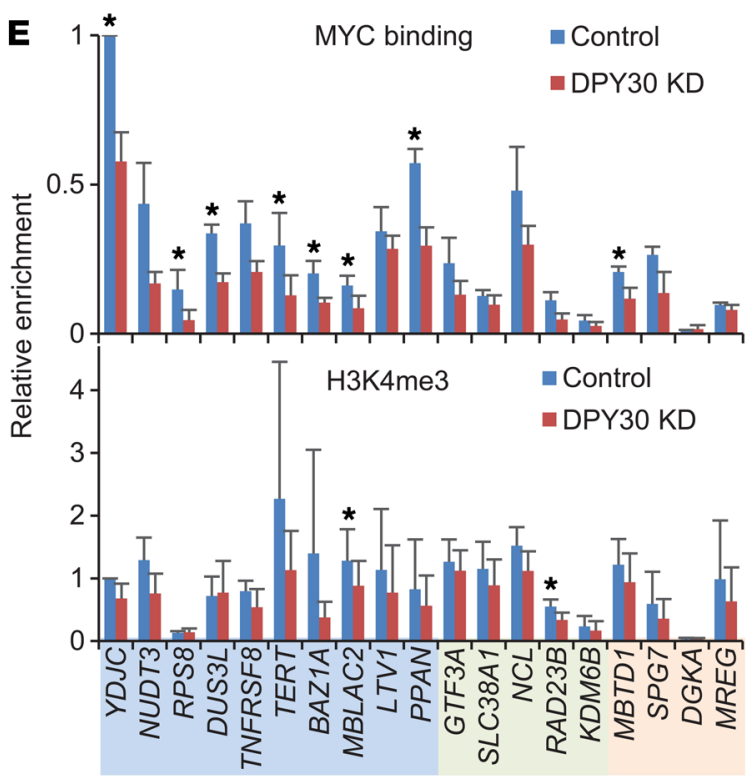

Figure 3. DPY30 is important for efficient binding of MYC to its genomic loci. (A) DPY30, MYC, and MAX mRNA levels were determined by $q P C R$ using 2 different primers for MYC and MAX and normalized to ACTB from 5 independent KD assays in P493-6 cells. (B) Total cell lysates from control and DPY30-KD P493-6 cells were used for immunoblotting with the indicated antibodies and for Ponceau $\mathrm{S}$ staining of histones. Graph shows the relative signal intensity, which was calculated as the ratio over GAPDH (for MYC) or histones (for H3K4me3) from 3 samples each. (C and D) Analyses of ChIP-seq from control and DPY30-KD P493-6 cells. (C) Composite profiles of MYC ChIP signals (top) at TSSs are grouped (and color coded) by the fold change upon DPY30 KD in P493-6 cells. There were 9,233, 9,457, and 33,451 genes in which MYC binding was reduced by more than 2-fold (MYC down >2), increased by more than 2-fold (MYC up >2), and changed by less than 2-fold in either direction (Others), respectively. The corresponding H3K4me3 ChIP signals are also shown for each group of genes. (D) MYC and H3K4me3 ChIP-seq profiles for representative genes within each group shown in $\mathbf{C}$. (E) Multiple genes within each group shown in $\mathbf{C}$ were validated by qPCR for MYC and H3K4me3 ChIP at TSSs, calculated from the ratio of the percentage of input value for each locus over that for the YDJC site in the control sample from 3 independent KD and ChIP assays in P493-6 cells. The results of individual repeats are also shown in Supplemental Figure 7. Data represent the mean $+\mathrm{SD}(\mathbf{A}, \mathbf{B}$, and $\mathbf{E}) .{ }^{*} P<0.05$, by Student's $t$ test.

turning MYC back on restored their expression (Figure 2D), further demonstrating the regulation of their expression by MYC. We performed this treatment on both control and DPY30-KD P493-6 cells and examined the global gene expression change after 4 hours of MYC reactivation, reasoning that such a short period of MYC reactivation mainly affects direct targets of MYC. We found that DPY30 KD significantly dampened the upregulation of most MYC-induced genes and also significantly impaired the downregulation of most MYC-repressed genes (Figure 2E and Supplemental Table 1). In other words, DPY30 loss blunted the transcription reprogramming mediated by MYC reactivation. Taken together, our results indicate that DPY30 functionally regulates the expression of MYC targets.
Dpy30 is important for efficient binding of MYC to its genomic loci. Unlike the regulation of endogenous MYC by DPY30 (Supplemental Figure 4B) $(28,31)$, expression of the tetracycline-controlled MYC transgene was not affected by DPYO KD at either the RNA or protein level in P493-6 cells (Figure 3, A and B). Moreover, we observed that the expression of $M A X$ was also not affected (Figure 3A). How, then, were MYC targets dysregulated following DPY30 KD?

We next assessed whether binding of MYC to its genomic targets was affected by DPY30 KD by performing MYC ChIP assays followed by both sequencing and quantitative PCR (qPCR) assays. We first confirmed the specificity of our MYC 


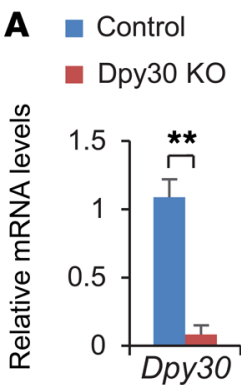

B
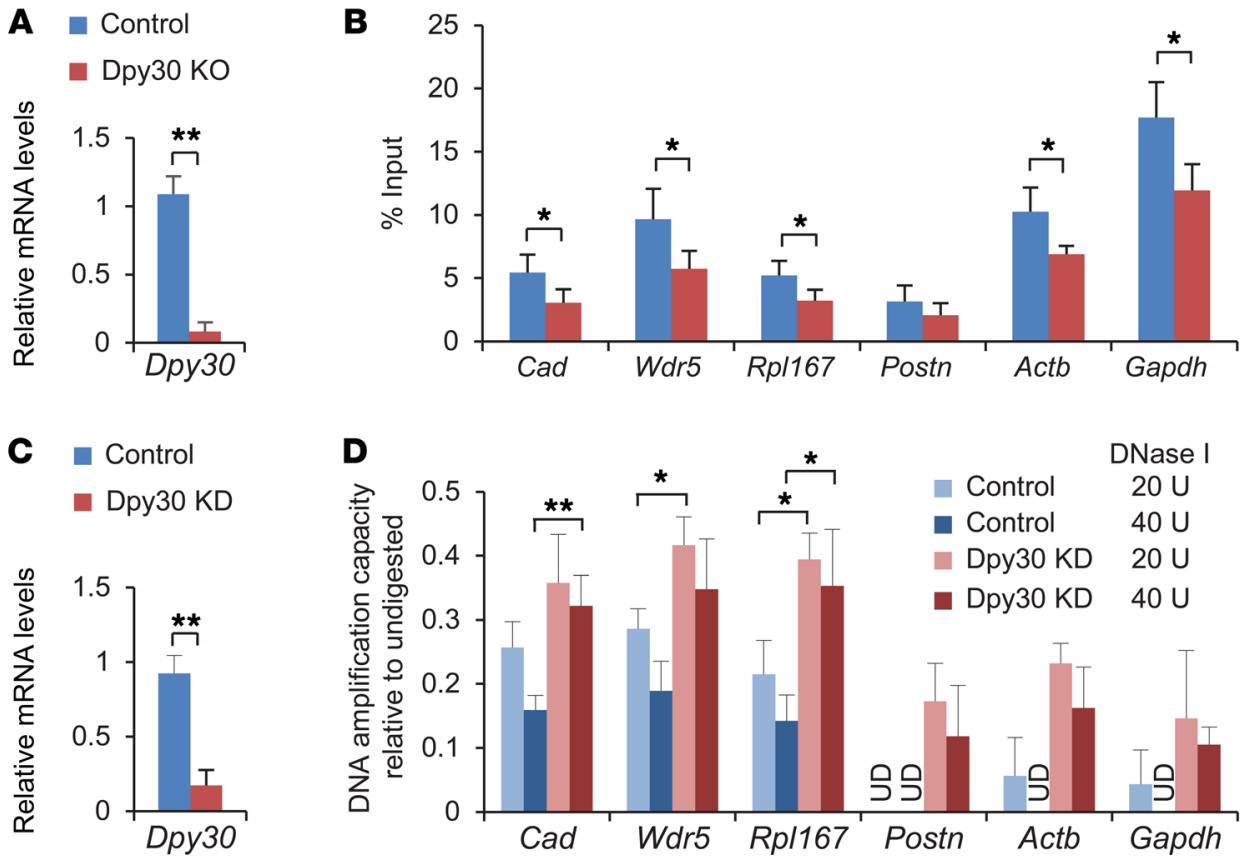

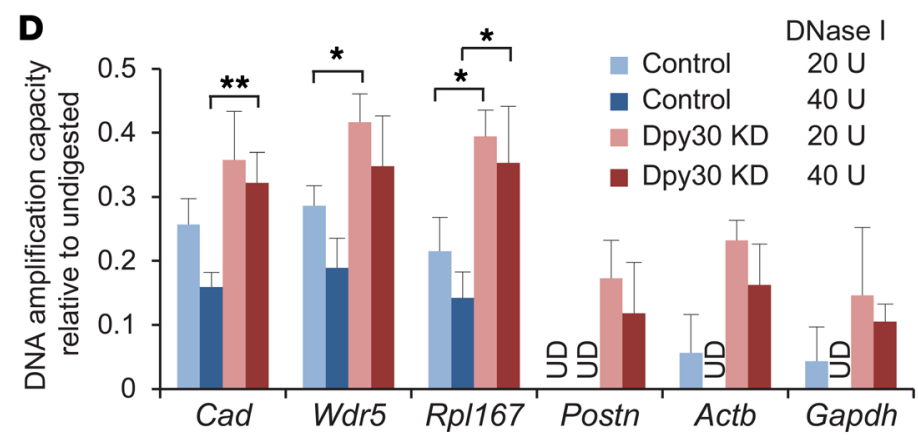

Figure 4. Dpy30 regulates chromatin accessibility. (A and B) Primary MEFs derived from $D p y 30^{f / / f l}$ (control)

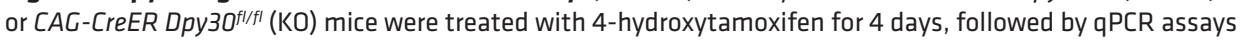
for relative mRNA levels of Dpy30, which was normalized to Gapdh (A), and by ATAC-qPCR (B). Results are from 4 independent 4-hydroxytamoxifen treatments followed by ATAC-qPCR assays. Related information is also provided in Supplemental Figure 9B. (C and D) Relative mRNA levels of Dpy30 normalized to Gapdh (C) and DNase I hypersensitivity assays (D) in MEFs expressing scrambled (control) or Dpy30 shRNA (KD). Results are shown as the mean + SD of triplicate measurements. In D, DNAs after digestion of 0, 20, or 40 units $/ \mathrm{ml}$ of DNase I were used for amplification of the indicated loci, and the amplification capacity of the digested samples relative to the undigested sample (set to 1 for both control and KD) was determined from triplicate measurements. Note that the higher the value, the more abundant the amplifiable DNA was after digestion, indicating less accessible chromatin. UD, undetectable by qPCR (suggesting high accessibility and complete digestion). Data represent the mean \pm SD. ${ }^{*} P<0.05$ and ${ }^{* *} P<0.01$, by Student's $t$ test $(\mathbf{A}-\mathbf{C})$ and 1 -factor ANOVA with a post hoc $t$ test (D).

antibody for ChIP by showing that MYC ChIP signals were dramatically reduced at all examined MYC targets after silencing MYC (Supplemental Figure 6A). Binding of MYC upon DPY30 KD was markedly reduced at a subset of gene transcription start sites (TSSs) and modestly reduced at the majority of TSSs (Figure 3C and Supplemental Table 2). While a small subset of genes showed an increase in MYC binding, they did not appear to be major MYC targets because of the very low MYC signals at their TSSs (Figure 3C and Supplemental Figure 6B). We also performed H3K4me3 ChIP assays. Consistent with previous findings $(12,13)$, there was a general correlation between MYC binding and H3K4me3 throughout the genome (Supplemental Figure 6C). H3K 4 me 3 was appreciably reduced at TSSs that showed reduced MYC binding upon DPY30 KD, but not at those that showed increased MYC binding (Figure 3, C and D; Supplemental Figure 6D; and Supplemental Table 3). We note that because the read counts in any specific regions were normalized to total read counts in the sample, the reduction seen on local MYC or H3K4me3 was most likely underestimated throughout the genome. Nevertheless, the relative effects for different gene loci were preserved. Our ChIP-qPCR assays confirmed a consistent decrease in MYC binding and H3K4me3 in several representative MYC target genes in all gene subsets, although the extent of the decrease differed somewhat among different subsets (Figure 3E and Supplemental Figure 7). We also examined the proliferation- or survival-associated genes that were downregulated by DPY30 KD in P493-6 cells and found that CCND2, CDCA7, $C D C A 5$, and BIRC5 were bound by MYC and that both MYC binding and $\mathrm{H} 3 \mathrm{~K} 4 \mathrm{me} 3$ at these genes were reduced upon DPY30 KD (Supplemental Figure 5F). Moreover, Dpy30 KD in MEFs (Supplemental Figure 6E) also markedly reduced Myc binding to a few established Myc targets (Supplemental Figure $6 \mathrm{~F}$ ), indicating that the effect of Dpy30 depletion on genomic binding of Myc is not limited to P493-6 cells.

We also studied the role of the ASH2L subunit, which directly binds to DPY30 and links it to the SET1-MLL complexes to facilitate H3K4 methylation (38). ASH2L is also amplified in many cancers (Supplemental Figure 1) and directly upregulated by activated MYC (Figure 1). As with DPY30 $\mathrm{KD}$, we found that ASH2L KD also abolished the growth of P493-6 cells (Supplemental Figure 8A), with little effect on tetracycline-controlled MYC expression (Supplemental Figure 8B). H3K4me3 was markedly reduced at almost all loci that we previously examined for DPY30 KD, and MYC binding was appreciably reduced at many of these loci (Supplemental Figure 8C). These results further support a role of the H3K4 methylation pathway in regulating MYC binding to its genomic targets.

To further understand how DPY3O and ASH2L regulate the genomic binding of MYC, we examined their physical interaction in in vitro binding assays. Consistent with previous reports (39), ASH2L directly binds to MYC. However, DPY30 failed to show direct binding with MYC (Supplemental Figure 9A). Therefore, DPY30 does not affect the activity of MYC through directly binding to MYC. As shown by assay for transposase-accessible chromatin using sequencing (ATAC-seq) (40) and DNase I hypersensitivity assays, we found that chromatin accessibility was reduced at assessed loci upon the reduction of Dpy30 and global H3K4me3 levels (Figure 4, A-D and Supplemental Figure 9B). Taken together, our results suggest that the Dpy30-regulated chromatin setting functionally influences the activity of MYC in transcription, prompting us to study the role of Dpy30 in Myc-driven tumorigenesis in vivo. 

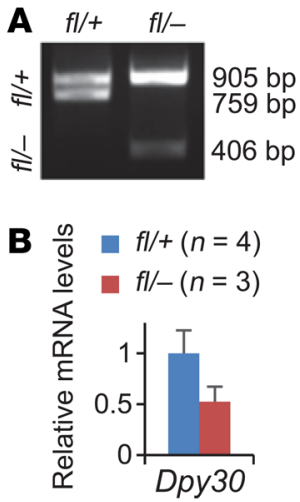

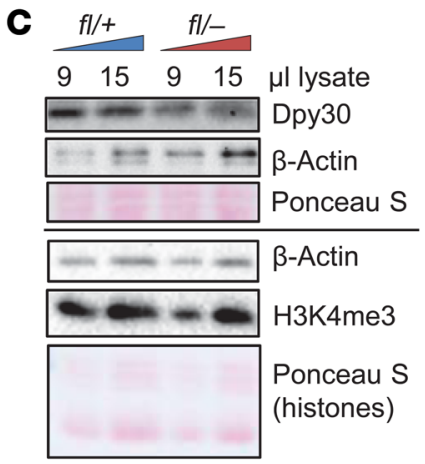

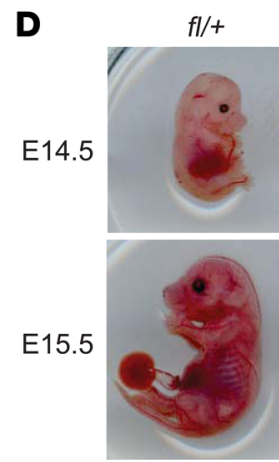
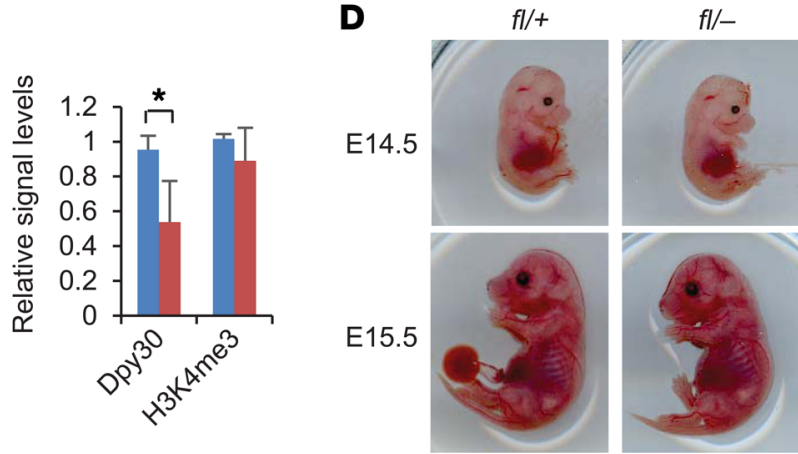

$\mathbf{E}$
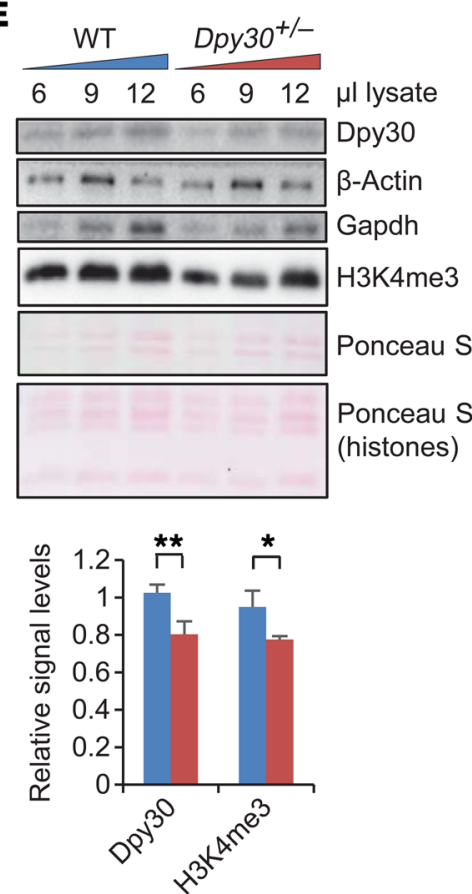

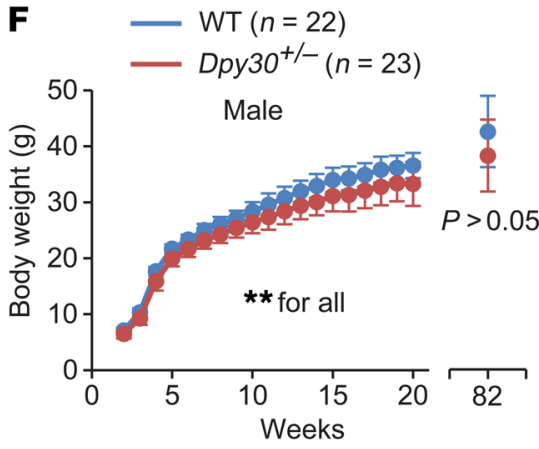

G

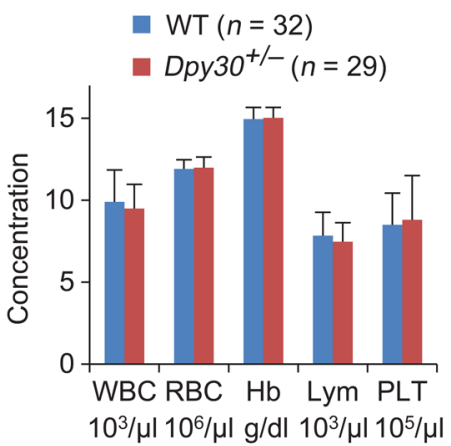

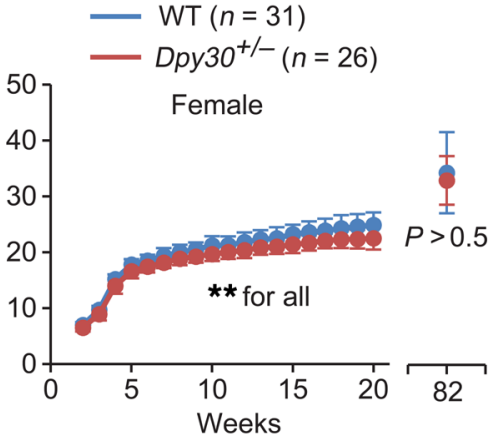

H

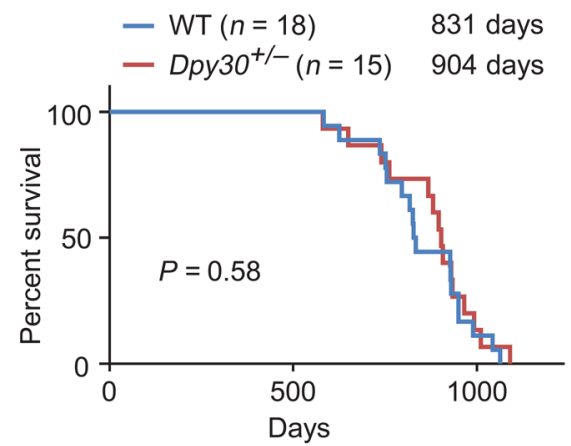

Figure 5. Dpy30 is haplosufficient for normal physiology of mice. (A) Results of genomic PCR to detect $D p y 30$ deletion in fetal livers of $D p y 30^{f / /+}$ and $D p y 30^{f /-}$ mice. The " $\mathrm{fl}$ " allele is the floxed (conditional) allele. The calculated sizes of the PCR products are shown on the right. (B) Relative mRNA levels of Dpy30 were determined by qPCR and normalized to Actb for Dpy3 $0^{f / /+}$ and Dpy3 $0^{f / /}$ fetal livers from the indicated numbers of E14.5 littermate embryos. (C) Immunoblot analysis for the indicated proteins (or modification), with increasing loading doses of lysates from fetal livers of $D p y 30^{f l /+}$ and $D p y 30^{f /-} E 14.5$ littermate embryos. Ponceau S stainings of irrelevant proteins and histones are also shown. Graph shows the relative signal intensity, which was calculated as the ratio over histones from 3 samples for each genotype at a single loading dose. (D) Gross morphology of Dpy30 f//+ and Dpy30 f//- embryos at E14.5 and E15.5. (E) Immunoblot for the indicated proteins (or modification), with increasing loading doses of lysates from splenic B cells of 4-week-old WT and Dpy30+- littermate mice. Ponceau S stainings of irrelevant proteins and histones are also shown. Graph shows the relative signal intensity, which was calculated the same ways as in C. (F) Body weight curves. The number of animals is indicated for all time points, except for week 82 , when $n=13$ for male WT mice; $n=15$ male $D p y 30^{+/-}$mice; $n=12$ female WT mice; and $n=7$ female $D p y 30^{+/-}$mice. (C) Peripheral blood cell and hemoglobin (Hb) concentrations for 8-week-old littermate mice. Lym, lymphocytes; PLT, platelets. (H) Kaplan-Meier curves. Data represent the mean $+\mathrm{SD}(\mathbf{B}, \mathbf{C}, \mathbf{E}$ and $\mathbf{G})$ or \pm SD (F). ${ }^{*} P<$ 0.05 and ${ }^{* *} P<0.01$, by Student's $t$ test ( $\mathbf{C}$ and $\mathbf{F}$, for each pair at each time point). The $P$ value in $\mathbf{H}$ was calculated by log-rank test.

Dpy30 is haplosufficient for normal animal development, physiology, and lifespan. As Dpy30 loss in the hematopoietic system results in pancytopenia (31), we first examined the effect of genetically reducing the Dpy30 dose on normal animal physiology. Compared with $D p y 30^{f /+}$ littermates ( $f$ denotes the conditional allele) (31), $D p y 30^{t /-}$ mouse embryos (Figure 5A) had an approximately 50\% reduction in Dpy30 expression at the RNA (Figure 5B) and protein (Figure 5C) levels, had slightly (and insignificantly) reduced glob- al H3K4me3 levels in their fetal livers cells (Figure 5C), and were indistinguishable in gross morphologic analyses (Figure 5D). Compared with the WT littermates, splenic B cells from $D p y 30^{+/}$mice also had an approximately 50\% reduction in Dpy30 expression at the RNA level (see below) and a modest reduction at the protein level (Figure 5E), as well as a modest reduction in global H3K4me3 levels (Figure 5E). Young, developing $D p y 30^{+-}$mice had slightly, albeit significantly lower body weights than did their WT littermates for 
A

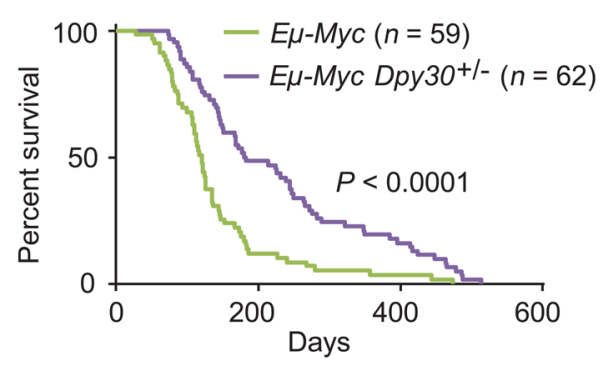

C
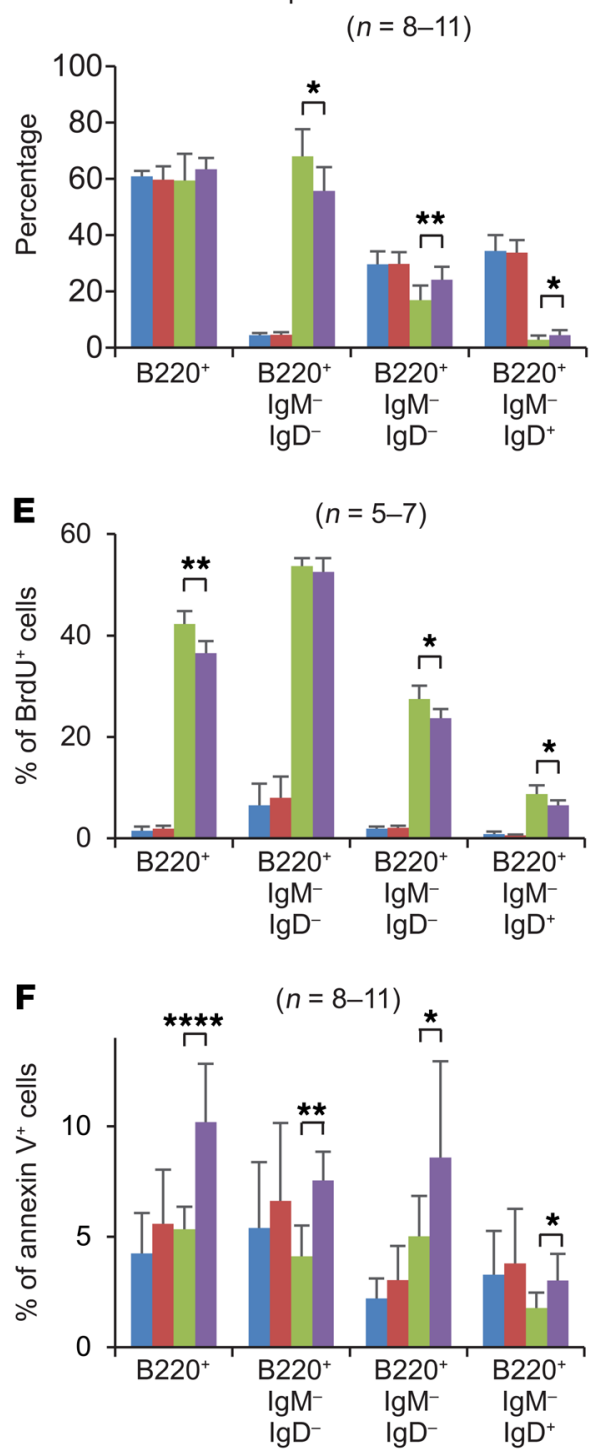

Median survival:

121 days

180.5 days
B

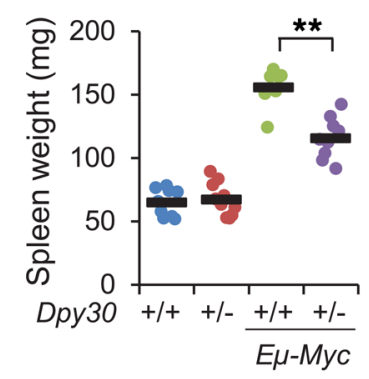

Bone marrow

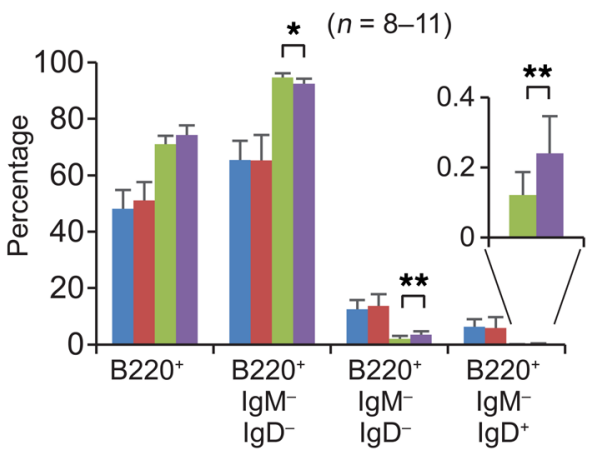

D

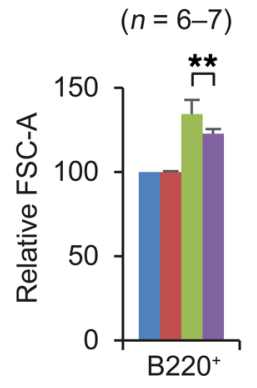

- WT

- Dpy $30^{+/-}$

E $\mathrm{n-Myc}$

- EM-Myc Dpy30+/-

$\mathrm{B} 220^{+}$
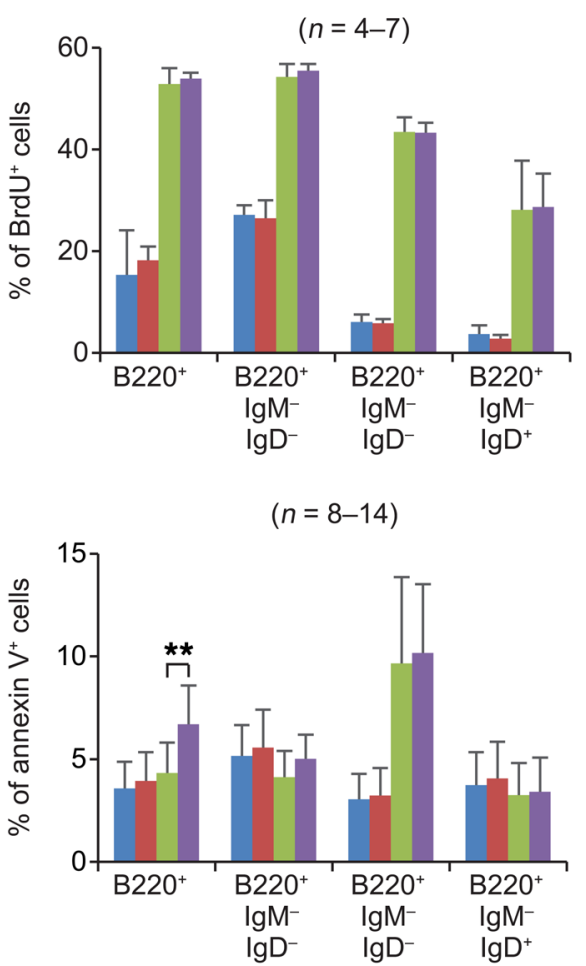

Figure 6. Dpy30 heterozygosity suppresses MYC-driven lymphomagenesis in mice. (A) Kaplan-Meier curves showing median survival. (B) Spleen weights

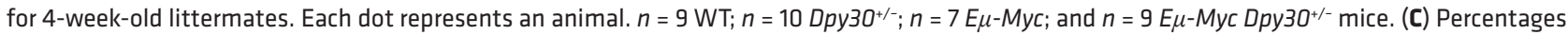
of $\mathrm{B}_{22 \mathrm{O}^{+}}$cells in total cells and percentages of the subpopulations in all B220+cells. For spleen and bone marrow each: $n=8 \mathrm{WT} ; n=11 \mathrm{Dpy} 3 \mathrm{O}^{+/-} ; n=11$ $E \mu-M y c$; and $n=8 E \mu-M y c$ Dpy30+/- cells. (D) Size of splenic B220+ cells as shown by the forward scatter area (FSC-A) values from FACS analysis. $n=6$ WT;

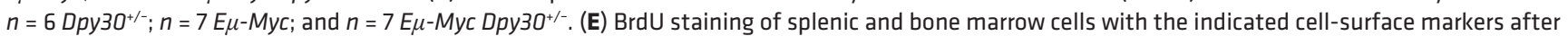

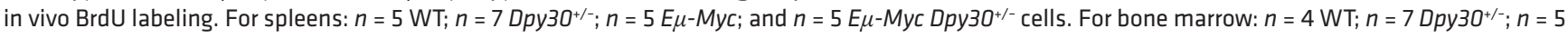
$E \mu-M y c ;$ and $n=5 E \mu-M y c$ Dpy30+/- cells. (F) Annexin V staining of splenic and bone marrow cells with the indicated cell-surface markers. For spleen: $n=8$

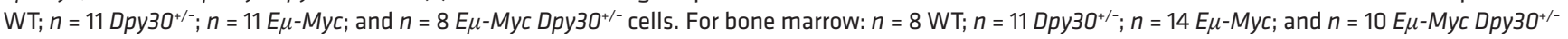
cells. Data represent the mean $\pm \mathrm{SD}(\mathbf{C}-\mathbf{F}) .{ }^{*} P<0.05,{ }^{* *} P<0.01$, and ${ }^{* * * *} P<0.0001$, by log-rank test $(\mathbf{A})$ and 1 -factor ANOVA with a post hoc $t$ test (B-F). 


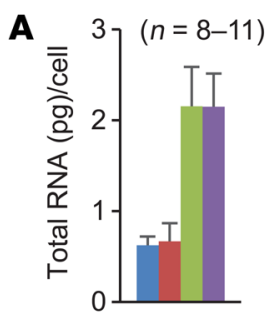

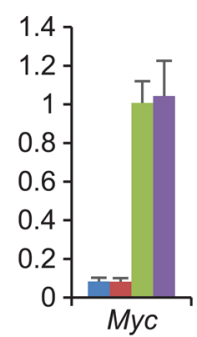

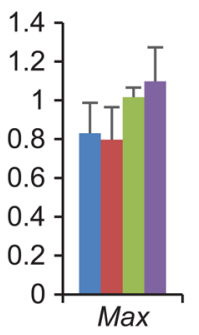

\author{
WT \\ Dpy30+/- \\ EM-Myc \\ EM-Myc Dpy30+/-
}

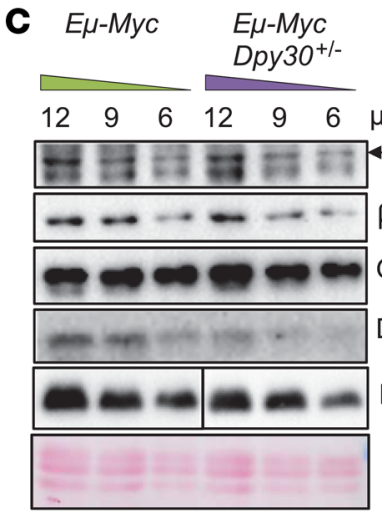

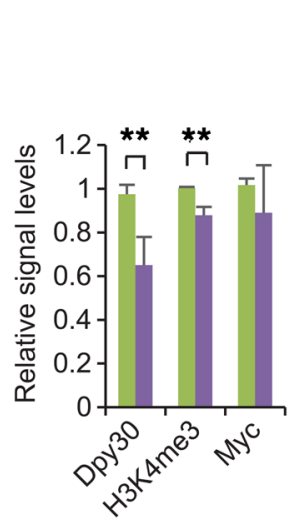

D

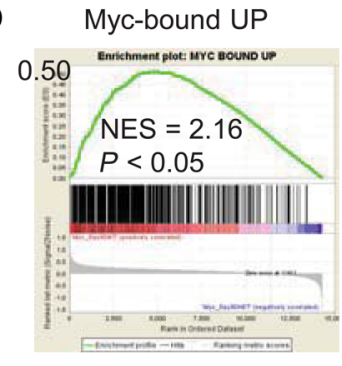

Myc-bound DN

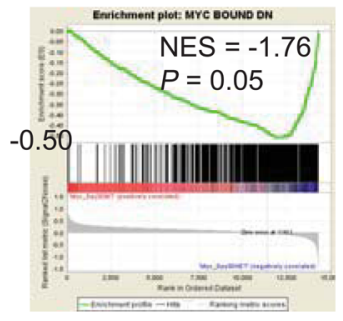

H3K4me3

Ponceau S (histones)
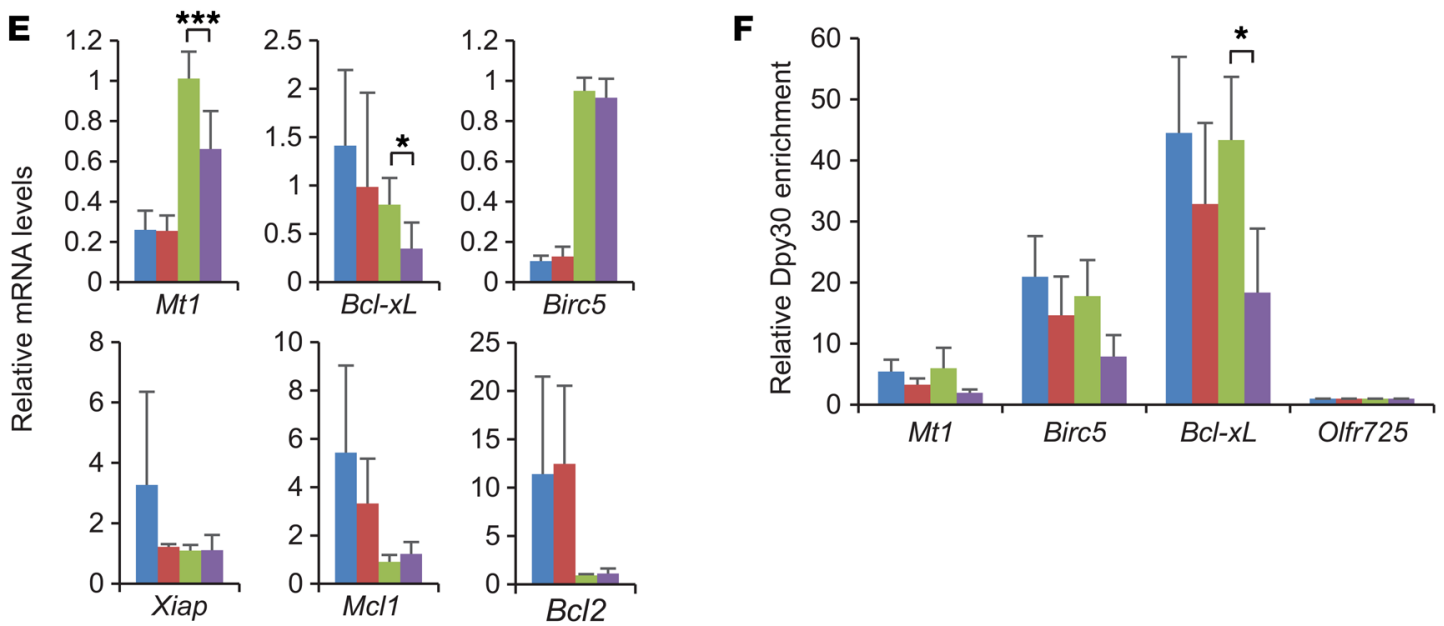

Figure 7. Impact of Dpy30 heterozygosity on gene expression in splenic B cells. All data are from purified splenic B220+ cells. (A) Total RNA levels per cell.

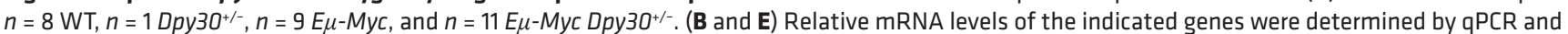
normalized to Actb, shown as the mean + SD. $n=6,6,8$, and 6 mice (for Dpy30 and $M y c$ in $\mathbf{B}$, and $B c l 2, B c l-x L$, and Birc5 in E); $n=5,4,9$, and 9 mice (for

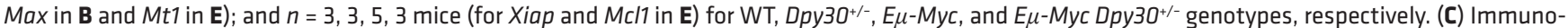
blot for the indicated proteins (or modification), with increasing loading doses of lysates. Graph shows the relative signal intensity, which was calculated as the ratio over $\beta$-actin and plotted from $3 \mathrm{E} \mu$-Myc or $4 \mathrm{E} \mu-\mathrm{Myc} D p y 3 \mathrm{O}^{+--}$samples at a single loading dose. (D) GSEA for the Myc-bound gene sets comparing gene expression profiles for $E \mu-M y c$ and $E \mu-M y c$ Dpy30+-- B cells. Myc targets that are most significantly upregulated (Myc-bound UP) or downregulated (Myc-bound DN) by Myc (curated from GEO GSE51011; see Methods and Supplemental Table 4) were used as gene sets, respectively. NES, normalized enrichment score. (F) Relative Dpy30 enrichment at the indicated gene TSSs was determined by Dpy30 ChIP on purified B220 ${ }^{+}$cells, calculated from the ratio of the percentage of input value for each locus over that for the negative control site (OIfr725) in E $\mu$-Myc samples from 3 mice of each genotype. Data represent the mean $+\mathrm{SD}$. ${ }^{*} P<0.05$, ${ }^{* *} P<0.01$, and ${ }^{* *} P<0.001$, by Student's $t$ test $(\mathbf{C})$ and 1 -factor ANOVA with a post hoc $t$ test $(\mathbf{A}, \mathbf{B}, \mathbf{E}$ and $\mathbf{F})$.

both sexes (Figure 5F) but appeared completely healthy, with a normal peripheral blood profile (Figure $5 \mathrm{G}$ ). Importantly, after following these animals for 3 years, we found that the $D p y 30^{+-}$mice had the same lifespan as their WT littermates (Figure 5H). Moreover, Dpy30 heterozygosity did not significantly affect the spleen weights or the development, size, proliferation, or apoptosis of the splenic or bone marrow B cells (Figure 6, B-F, and Supplemental Figure 10A). We detected no significant alteration of cellular damage signals, such as the DNA damage response (Supplemental Figure 10B) or ROS levels (Supplemental Figure 10C), following inactivation of 1 Dpy30 allele. Therefore, we conclude that a full level of Dpy30 is largely dispensable for normal animal physiology.

Dpy30 heterozygosity suppresses Myc-driven lymphomagenesis. We then studied the effects of Dpy30 reduction in the $E \mu-M y c$ lymphomagenesis mouse model. We found that $E \mu-M y c D p y 3 O^{+/-}$mice survived significantly longer than did their $E \mu-M y c$ littermates, with an 
A
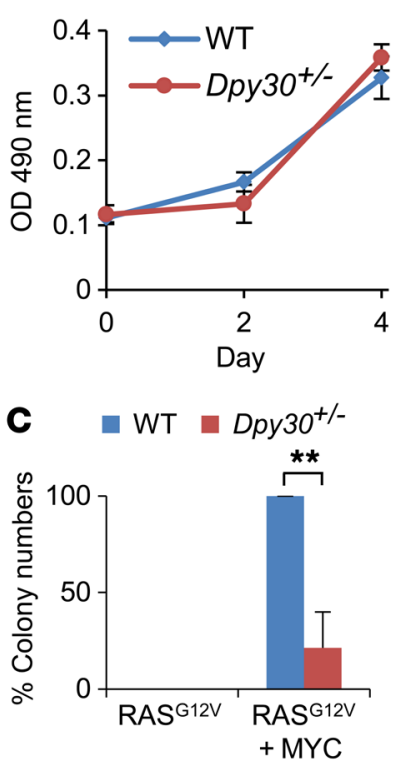

B

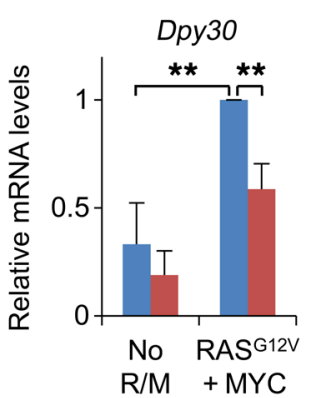

- WT $\square$ Dpy30 ${ }^{+/-}$
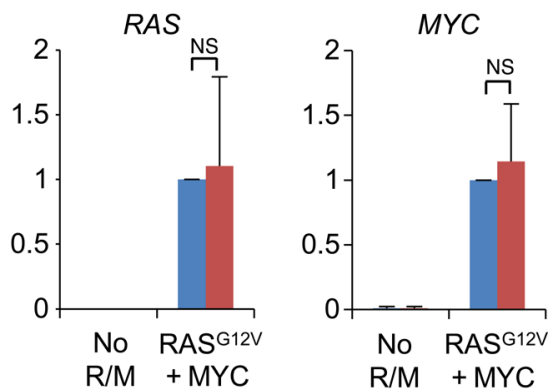

D
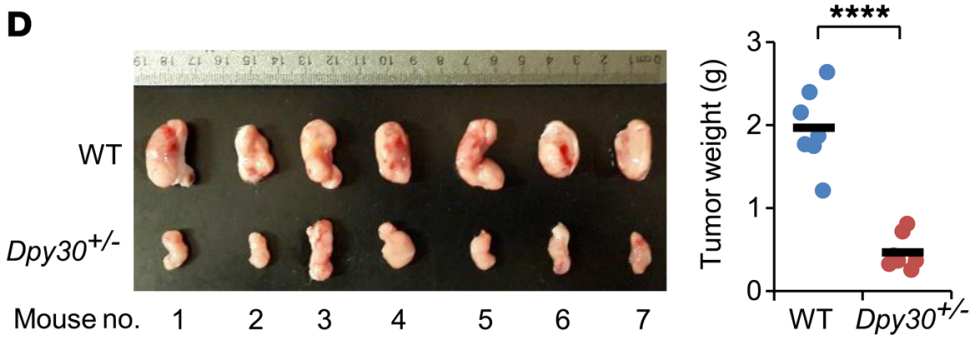

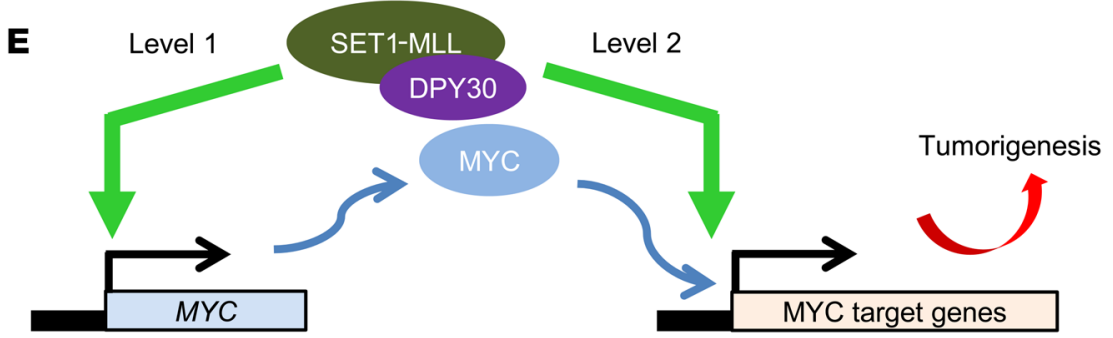

Figure 8. Dpy30 is haploinsufficient for oncogenic transformation but not for cell growth. (A) WT and $D p y 30^{+/-} \mathrm{MEFs}$ were assayed for growth, shown as the MTT assay results from 3 biological replicates. (B and C) Representative results of soft agar colony formation assay for HRAS ${ }^{\mathrm{G} 12 \mathrm{~V}}$ and MYC-mediated oncogenic transformation of WT and Dpy30/- MEFs. (B) Relative mRNA levels of DPY30, RAS, and MYC in untransduced MEFs and MEFs transduced with HRAS $^{\mathrm{G} 12 \mathrm{~V}}$ and MYC viruses were determined by qPCR and normalized to Actb from the 3 independent transformation assays shown in $\mathbf{C}$ and $\mathbf{D}$. The levels in WT MEFs infected with HRAS ${ }^{\mathrm{G} 12 \mathrm{~V}}$ and MYC were set to 1. (C) The percentage of colony numbers relative to WT (mean number was 59 for WT) was determined from 3 independent transformation assays using 2 different embryo-derived MEF samples for each genotype. (D) WT and Dpy30+/- MEFs transduced with $\mathrm{HRAS}^{\mathrm{G} 12 \mathrm{~V}}$ and MYC viruses were injected into the flanks of 7 NSG mice. Each mouse received WT MEFs in $1 \mathrm{flank}$ and the $D p y 30^{+/-}$MEFs in the other flank. Two weeks after injection, tumors were collected (shown) and weighed. The tumor weights were plotted, and each dot represents a tumor from an animal. (E) Model illustrating the 2 different levels of regulation of MYC by DPY30 complexes. Data represent the mean \pm SD (A) or + SD (B and $\mathbf{C})$. ${ }^{*} P<0.01$ and ${ }^{* * *} P<0.0001$, by 1 -factor ANOVA with a post hoc $t$ test (B) and Student's $t$ test (C and $\left.\mathbf{D}\right)$.

increase of nearly $50 \%$ in the median survival time (Figure $6 \mathrm{~A}$ ) and significantly mitigated MYC-driven spleen enlargement (Figure 6B). These results demonstrate that reducing Dpy30 levels suppresses MYC-driven lymphomagenesis without affecting normal physiology.

We found that Dpy30 heterozygosity modestly but significantly mitigated the developmental block from pre-B to mature B cells and the cell size increase caused by the $E \mu-M y c$ transgene (Figure 6, C and D; and Supplemental Figure 10A). E $\mu-M y c$ activation dramatically increased the proliferation of all $\mathrm{B}$ cells in the spleen and bone marrow. We found that $D p y 30$ heterozygosity modestly but significantly reduced the proliferation of certain subpopulations of $\mathrm{B}$ cells in the spleen (Figure 6E). All these results are consistent with reduced MYC activity in B cells upon inactivation of 1 Dpy30 allele.

We next examined B cell apoptosis. Although Myc hyperactivation can elicit apoptosis of human fibroblasts (41), we detected no significant increase in apoptosis of splenic or bone marrow B cells in $E \mu-M y c$ mice with intact Dpy30 levels, except the
$\mathrm{B}^{200^{+}} \mathrm{IgM}^{+} \mathrm{IgD}^{-}$subpopulation in bone marrow (Figure $6 \mathrm{~F}$ ). When 1 Dpy30 allele was inactivated, however, we found that apoptosis was significantly and markedly increased for $E \mu-M y c$ B cells at all developmental stages in spleen and for the overall B cell populations in bone marrow (Figure 6F). Therefore, cellular stresses imposed by $M y c$ hyperactivation would have elicited strong apoptosis, had it not been for a sufficient level of Dpy30 and its enforced elevation by Myc (further shown and discussed below).

Since Dpy30 depletion in human fibroblasts results in an increase in the DNA damage response and ROS (29), we examined whether these also occur in Dpy30 heterozygous B cells. We found that, while the total levels of phosphorylated H2AX $(\gamma-\mathrm{H} 2 \mathrm{AX})$ and ROS were markedly increased in the splenic B cells of $E \mu-M y c$ compared with WT mice, Dpy30 heterozygosity did not affect the levels of these signals or total p53 (Supplemental Figure 10, B and C). Therefore, $D p y 30$ heterozygosity does not induce apoptosis by increased DNA damage or ROS. 
Effect of Dpy30 heterozygosity on gene expression in B cells at different malignant stages. We next determined the impact of Dpy30 heterozygosity on gene expression in B lymphocytes in the absence and presence of the $E \mu-M y c$ transgene, using B200+ splenocytes from nontransgenic control mice and premalignant $E \mu-M y c$ mice. The total RNA amount per cell was dramatically increased by $E \mu-M y c$ expression but was not affected by Dpy30 heterozygosity in B cells, regardless of the transgene (Figure 7A). Dpy30 expression was upregulated by over 2-fold by $E \mu-M y c$, reduced to half by inactivation of $1 D p y 30$ allele, either in the absence or presence of $E \mu-M y c$, and was similar between WT and $E \mu-M y c D p y 30^{+-} \mathrm{B}$ cells (Figure 7B). The expression of Myc or Max was not significantly affected by Dpy 30 heterozygosity (Figure 7, B and C). We observed that global H3K4me3 levels were modestly reduced in $E \mu-M y c$ Dpy30 ${ }^{+/-}$compared with levels in $E \mu-M y c$ B cells (Figure 7C). Using spike-in RNA control, our RNA-sequencing (RNA-seq) analyses followed by gene set enrichment analysis (GSEA) (42) showed that the genes downregulated in $E \mu-M y c p y 30^{+/-}$compared with $E \mu-M y c$ $B$ cells were significantly enriched with genes bound and activated by Myc (Figure 7D left, Supplemental Figure 11 and Supplemental Tables 4 and 5) and that the upregulated genes were enriched with genes bound and repressed by Myc (Figure 7D right, Supplemental Figure 11 and Supplemental Tables 4 and 5). Moreover, our gene ontology analyses revealed that genes downregulated by $D p y 30$ heterozygosity in the presence of $E \mu-M y c$ were enriched with common Myc targets such as genes in ribosome and RNA biogenesis (Supplemental Figure 12A and Supplemental Table 6). These results in animals are consistent with those obtained in P493-6 cells, in which the expression of Myc targets, but not $M y c$ itself, was dysregulated upon Dpy30 loss.

Consistent with the increased apoptosis, the expression of cytotoxic genes such as FasL and Gzmb was significantly upregulated (Supplemental Figure 12B and Supplemental Table 6), while the expression of a subset of prosurvival genes such as $M t 1$ $(43,44)$ and $B c l-x L(45)$ was significantly downregulated in the $E \mu-M y c D p y 30^{+-}$compared with $E \mu-M y c$ B cells (Figure 7E). We found that Dpy30 bound to the TSSs of Mt1, Birc5, and Bcl- $x L$ genes in splenic B cells (Figure 7F), suggesting a direct regulation of these prosurvival genes by Dpy30, although the extent of the consequence on their expression varied upon Dpy30 reduction. A further dissection around the $M t 1$ TSS region showed that, consistent with the induction of $M t 1$ by $E \mu-M y c$ (Figure 7E), Dpy30 was increasingly recruited to the $M t 1$ TSS following $E \mu-M y c$ activation, resulting in enhanced H3K4 methylation (Supplemental Figure 13). Upon inactivation of 1 Dpy30 allele in the presence of the $E \mu-M y c$ transgene, promoter binding of Dpy30 was reduced, leading to reduced H3K4me3 (Supplemental Figure 13) and inefficient expression of $M t 1$. These results suggest that MYC promotes Dpy30 to ensure sufficient expression of certain prosurvival genes to suppress an otherwise detrimental apoptotic response to cancer cells.

To understand how lymphomas eventually formed in the $E \mu-M y c D p y 30^{+/-}$mice, we examined the expression of all subunits of Set1-Mll complexes and prosurvival genes in these tumor samples. We found that Dpy30 expression in E $\mu-M y c D p y 30^{+-}$ lymphomas was still reduced to approximately $50 \%$ at the RNA level and markedly reduced at the protein level compared with expression levels in $E \mu$-Myc lymphomas (Supplemental Figure 14). The expression levels of all other catalytic and core subunits of the Set1-Mll complexes and of Myc were not significantly altered (Supplemental Figure 14). While $B c l$ - $x l$ expression was significantly reduced, we found that $M t 1$ expression was no longer affected by Dpy30 heterozygosity in lymphomas (Supplemental Figure 14A). These results suggest that an altered regulation of selected Dpy30 targets may allow bypass of the Dpy30 pathway and underlie the ultimate formation of tumors, despite the reduction of Dpy30.

Dpy30 is haploinsufficient for cellular transformation but not for normal growth. To determine whether the differential requirement of Dpy30 dose is limited to $E \mu-M y c$-driven lymphomagenesis, we studied the impact of Dpy30 heterozygosity on the transformation of primary MEFs by 2 potent oncogenes, $H-R A S^{G 12 V}$ and MYC. Dpy3O heterozygosity reduced Dpy3O mRNA levels by approximately 50\% (Figure $8 \mathrm{~B}$ ) and did not significantly affect the growth of primary MEFs (Figure 8A). DPY30 expression was upregulated by approximately 3-fold following $\mathrm{H}-\mathrm{RAS}^{\mathrm{G} 12 \mathrm{~V}}$ and MYC transduction of the WT MEFs (Figure 8B), but not after transduction of H-RAS ${ }^{\mathrm{G} 12 \mathrm{~V}}$ alone (data not shown), further indicating its regulation by Myc. Again, Dpy30 levels were reduced to approximately $50 \%$ in transduced $\mathrm{Dpy} 3 \mathrm{O}^{+/-} \mathrm{MEFs}$, but were still higher than those in untransduced WT MEFs (Figure 8B). We also determined that the expression levels of both oncogenes were comparable between WT and Dpy30+/- MEFs (Figure 8B). As reflected by the anchorage-independent colony formation, the WT MEFs were efficiently transformed by these oncogenes. We found that transformation of $D p y 30^{+/-} \mathrm{MEFs}$, however, was dramatically impeded (Figure $8 \mathrm{C}$ and Supplemental Figure 15A). In vivo tumorigenicity of the transduced MEFs was also significantly reduced by Dpy30 heterozygosity (Figure 8D). While proliferation was similar between WT and $D p y 30^{+/-} \mathrm{MEFs}$ following RAS-MYC transduction, we found that apoptosis (and general cell death) was significantly higher in $D p y 30^{+/-}$MEFs compared with WT MEFs after transduction (Supplemental Figure 16), consistent with the observations in $E \mu-M y c$ B cells. These results reveal a contrasting requirement of the Dpy30 dose. These results reveal a contrasting requirement of Dpy30 dose - while the regular Dpy30 level is more than enough for normal cell growth, it must be elevated for efficient cancerous transformation by oncogenes, possibly through combating oncogene-associated apoptotic stress.

We next assessed the direct consequences of overexpression of the SET1-MLL complex core subunits in cellular transformation. A previous study (24) has shown that primary rat embryonic fibroblasts can be transformed by overexpressing RAS ${ }^{\mathrm{G} 12 \mathrm{~V}}$ and ASH2L. Here, we found that MEFs that overexpress RAS ${ }^{\mathrm{G} 12 \mathrm{~V}}$, together with either DPY30 or ASH2L, formed colonies in soft agar, although the number of colonies was smaller than that induced by RAS ${ }^{\mathrm{G} 12 \mathrm{~V}}$ and MYC (Supplemental Figure 15B). Interestingly, while the combination of RAS ${ }^{\mathrm{G} 12 \mathrm{~V}}$ with ASH2L or DPY30 gave rise to a similar number of total colonies, $\mathrm{RAS}^{\mathrm{G} 12 \mathrm{~V}}$ with $\mathrm{ASH} 2 \mathrm{~L}$ did not give rise to a large number of colonies like RAS ${ }^{\mathrm{G} 12 \mathrm{~V}}$ with DPY30 or with MYC (Supplemental Figure 15B). These results indicate that DPY30 and ASH2L can each individually cooperate with RAS ${ }^{\mathrm{G} 12 \mathrm{~V}}$ to promote transformation. The similar phenotype suggests that these proteins regulate a common pathway (presumably Set1-Mll complex- 
mediated $\mathrm{H} 3 \mathrm{~K} 4$ methylation) in promoting transformation, but the difference in the colony size suggests that Dpy30 may regulate additional pathways in the control of transformation.

\section{Discussion}

Our studies reveal a functional relationship between a major epigenetic mechanism and an important oncogene. We have previously shown that DPY30 directly regulates the expression of endogenous MYC in hematopoietic cells $(28,31)$. Here, we reinforced this conclusion in other MYC-overexpressing blood cancer cells and show that MYC also directly regulates the expression of several core subunits, including DPY30, of the major H3K4 methyltransferase complexes in several cellular systems. Therefore, DPY30 and MYC mutually and positively regulate each other. The direct upregulation of DPY3O by MYC is functionally important, as insufficient DPY30 levels clearly impair MYC-driven lymphomagenesis and cellular transformation.

Although H3K4 methylation is well known to be intimately associated with active transcription and biochemically capable of directly enhancing chromatin transcription, its biological functions in physiology and pathology are surprisingly murky, partly because of the complex composition and functions of the many subunits of the complexes collectively responsible for the enzymatic activity. Most of the core subunits of SET1-MLL complexes are considered essential for the efficient H3K4 methylation activity of all 6 catalytic subunits (46), and their expression levels are thus generally expected to be limiting for gene expression in the cells and physiology of animals. Our results here show that the Dpy30 subunit exists in excess for normal physiology from embryonic development throughout the lifespan of the animal, raising the question of why this subunit is maintained at a surplus level. One possible explanation is its potential role in handling stressful conditions. In this work, we show that oncogenic activation, such as MYC overexpression, not only co-opts existing excessive DPY30 levels, but also directly and selectively upregulates its expression (and other SET1-MLL complex core subunits) in order to play a critical role in tumorigenesis. Such upregulation is necessary, because a Dpy30 level similar to or just modestly higher than that in normal cells (as seen in the MYC-overexpressing $D p y 30^{+/-} \mathrm{B}$ cells and MEFs, Figure $7 \mathrm{~B}$ and Figure $8 \mathrm{~B}$ ) is insufficient for tumorigenesis. Therefore, the hyperactivated MYC oncogene, in a sense, has evolved to hijack key chromatin modulators to promote tumorigenesis.

The selective regulation of the core, but not the catalytic, subunits of the SET1-MLL complexes by MYC is consistent with the divergent alterations and possibly divergent roles of these subunits in human cancers. Indeed, mutations (mostly loss-of-function) in genes encoding the catalytic subunits of the SET1-MLL complexes are among the most frequent genetic lesions in human cancers (19-21, 23, 26, 47, 48). Moreover, the tumor-suppressive roles of some of these subunits have been demonstrated $(49,50)$. On the other hand, genetic mutations of the core subunits are rarely found in human cancers. Instead, the DPY30 (51), ASH2L $(24,52)$, and WDR5 $(25,53,54)$ core subunits have been found to be overexpressed in cancers, show correlation of high expression levels with poor prognosis, and, in some cases, functionally promote tumorigenesis. Such differential regulations and roles elicit interesting questions relating to the exact roles of these subunits and the associated H3K4 methylation in cancer and merit further investigation.
Our previous and current studies have collectively uncovered 2 different levels of regulation of MYC activity by DPY30 (Figure 8E). First, DPY30 controls MYC expression (28, 31). Second, DPY30 regulates the efficient binding of the MYC oncoprotein to its genomic targets. This advances our understanding from mainly correlative observations to that of the functional role of $\mathrm{H} 3 \mathrm{~K} 4$ methylation for efficient MYC binding to the genome. Targeting DPY30, which inhibits the crucial activity of the MYC oncoprotein, might offer an alternative strategy for treating cancers that evade the inhibition of MYC expression following other treatments, such as those involving the impressive BET protein inhibitors that repress $M Y C$ expression (55) but can induce resistant cancer cells with restored MYC expression $(56,57)$.

Our results show divergent effects of DPY30 reduction on MYC-mediated gene expression in both P493-6 and splenic B cells, reminiscent of the divergent impact on the induction and suppression of transcription changes during retinoic acid-mediated differentiation of mouse embryonic stem cells (27). It has been a longstanding observation that, despite the prevalent association of active chromatin marks with transcription, perturbing active chromatin marks can lead to changes in the expression of target genes in both directions. The underlying mechanisms remain poorly understood, other than the common interpretation of indirect effects. Our results here suggest that DPY3O and efficient H3K4 methylation may help maintain a promoter environment more accessible for the binding of transcription factors to exert either an activating or repressive effect, rather than strictly promoting active transcription. This mechanism may be applicable to other chromatin modulators as well.

The precise molecular mechanisms by which DPY30 regulates the genomic binding of MYC remain unclear. Although H3K4 methylation is generally thought to be a permissive chromatin mark, the direct measurement of chromatin accessibility change upon perturbation of this modification has not to our knowledge been reported. Our results suggest that DPY3O and efficient H3K4 methylation are important for maintaining the high chromatin accessibility of the tested loci and together provide a possible mechanism for their role in the genomic binding of MYC. A quantitative assessment of genome-wide chromatin accessibility was unsuccessful because of the difficulty of normalization across samples in which signals throughout the genome are likely to be affected (thus lacking the internal reference loci). Future work will need to improve the quantitative assessment to address this important mechanistic question. A general impact on chromatin accessibility suggests that DPY30 levels may regulate the binding of transcription factors beyond MYC, which is supported by our previous finding that Dpy30 is important for exogenous Oct4 to bind to its chromatin targets during cellular reprogramming (30). However, being a non-pioneer factor $(13,58)$, MYC is probably exceptionally dependent on the permissive chromatin environment facilitated by high DPY30 levels.

Although DPY30 is not essential for the integrity of the SET1MLL complexes (59) and does not directly bind MYC, we cannot exclude the possibility that a reduction in DPY30 may indirectly affect the binding of MYC with ASH2L, WDR5, or BPTF. These proteins physically associate with DPY30 (15) and regulate genomic binding of MYC $(60,61)$. Although a previous study showed that modest depletion of ASH2L did not affect genomic binding of MYC to a few target genes (39), we found here that ASH2L KD reduced 
MYC binding at many gene loci (Supplemental Figure 8C). Interestingly, while DPY30 KD had a stronger effect than ASH2L KD did on MYC binding throughout these loci, we observed a stronger effect on $\mathrm{H} 3 \mathrm{~K} 4 \mathrm{me} 3$ at these loci as a result of ASH2L KD. These results suggest that DPY30 probably regulates genomic recruitment of MYC, in part via its role in ASH2L-SET1-MLL complexes and also in part via its interaction with other factors, such as BPTF in the NURF chromatin-remodeling complex (15). The general importance of the core subunits of the SET1-MLL complexes in promoting the binding of MYC to its genomic targets may underlie the general role of these subunits in oncogenesis, befitting their frequent amplification in cancers. In addition to regulating MYC binding to the genome, DPY3O and H3K4 methylation can modulate the recruitment of H3K4 methylation readers (62) to regulate the transcription of both MYC and non-MYC targets. Moreover, DPY30 may directly regulate the recruitment of BPTF and the NURF complex via physical association $(15,63)$. Indeed, some genes that are not MYC targets were also altered in the $E \mu-M y c D p y 30^{+/-}$cells compared with that observed in $E \mu-M y c$ B cells (Supplemental Figure 11) and may also contribute to their reduced tumorigenesis.

High Dpy30 levels ensure sufficient expression of a subset of prosurvival genes to counteract the apoptotic effects brought on by the oncogenic assault. We show that the expression of $M t 1$ and $B c l-x L$, but not $B c l 2$, was significantly downregulated in $E \mu-M y c$ Dpy30 ${ }^{+/-} \mathrm{B}$ cells compared with expression levels in the $E \mu-M y c$ littermate $\mathrm{B}$ cells. Considering the specific requirement of endogenous $B c l-x L$ (64), but not Bcl2 (65), for Myc-driven lymphomagenesis in mice, these results suggest a possible functional contribution of $B c l-x L$ regulation by Dpy30 in MYC-driven lymphomagenesis.

The role of high Dpy30 levels in combating apoptotic stress is also seen in primary MEFs following oncogene transduction. In the P493-6 BL cell model, however, DPY30 primarily promoted MYC-driven proliferation, with little effect on apoptosis. This is probably a collective result of the modest downregulation of several proliferation-related genes (Supplemental Figure 5E). CCNA2 and MCM1O have either unaffected MYC binding or very little MYC binding (Supplemental Figure 5F), and their downregulation by DPY30 KD may be a direct consequence of reduced H3K4 methylation, presumably followed by reduced recruitment of H3K 4 methylation readers involved in transcription (62), as discussed above. These results suggest that DPY30, being a global epigenetic modulator, can exert differential effects on functional targets in different cellular systems.

Our data overall support the notion that the prominent epigenetic pathway of H3K4 methylation may be hijacked by oncogenes to promote tumorigenesis. Meanwhile, hyperactivation of oncogenes such as MYC renders tumor cells more dependent than normal cells are on DPY30 (and probably other core subunits of the SET1-MLL complexes) for survival. Such a differential dependence provides a basis for the increasingly observed but poorly understood "epigenetic vulnerability" of certain cancers (66), which can be exploited for potential cancer treatment.

\section{Methods}

Cell culture, gene KD, growth, and transformation assays. P493-6 cells (37) were a gift from Alanna Ruddell (Fred Hutchinson Cancer Research Center, Seattle, Washington, USA), with the permission of
Dirk Eick (Helmholtz Center, Munich, Germany). Raji and Jurkat cells were a kind gift from Tim Townes (University of Alabama at Birmingham). All of these cells were cultured in RPMI 1640 medium with $10 \%$ FBS. Primary MEFs were isolated from E13.5 embryos, cultured in MEF culture medium (DMEM supplemented with L-glutamine, 10\% FBS, $0.1 \mathrm{mM}$ nonessential amino acids, and $55 \mu \mathrm{M} \beta$-mercaptoethanol, all from Invitrogen, Thermo Fisher Scientific), and the passage number was kept to a minimum. Transformations were performed on early-passage cells (P3 or earlier). Retroviral particles were produced by transfecting $293 \mathrm{~T}$ cells with pWZL-c-MYC-bsd (Addgene, plasmid no. 10674) or pBabe-HRas-V12-puro (Addgene, catalog 1768) and an ecotropic packaging vector using Polyethylenimine (PEI) Transfection Reagent (Polysciences Inc.). Viral supernatants were filtered through a 0.45- $\mu \mathrm{M}$ filter. MEFs were infected with HRAS-V12 and C-MYC viral particles followed by selection in puromycin $(2 \mu \mathrm{g} / \mathrm{ml})$ and blasticidin $(2 \mu \mathrm{g} / \mathrm{ml})$. Soft agar colony formation assays were performed by plating transformed MEFs in a 24-well plate at 2,000 cells/well. MEFs were cultured in a layer of MEF culture medium in $0.3 \%$ agar over a base layer composed of culture media in $0.6 \%$ agar and fed every 4 days. Colonies were formed over the course of 3 to 4 weeks. For stable KD, P493-6 cells or MEFs were infected with lentiviruses expressing control or DPY30 shRNAs (all sequences are available in ref. 28), followed by puromycin $(2 \mu \mathrm{g} / \mathrm{ml})$ selection for 2 to 3 days starting 2 days after infection. Growth assays were performed in the absence of selecting antibiotics to avoid possible effects by differential expression of the antibiotic-resistant gene. P493-6 cell growth was measured by manual counting, and MEF cell growth was determined using a CellTiter 96 AQueous One Solution Cell Proliferation Assay (Promega, G3580).

Mice and tumor analyses. All mice were maintained under specific pathogen-free conditions and housed in individually ventilated cages. The $D p y 3 \mathrm{O}^{+-}$mouse was generated in our laboratory before, and the genomic PCR assays and the primers used were previously reported (31). $E \mu-M y c$ mice were purchased from The Jackson Laboratory (stock no. 002728). Breeding was set up to generate WT, Dpy30 $0^{+/}, E \mu-M y c$, and $E \mu-M y c D p y 3 O^{+/-}$littermate mice. Peripheral blood profiles were measured using a Hemavet 950 system (Drew Scientific). The mice were monitored for illness and tumor development. Terminally sick mice were humanely sacrificed, and tumors were collected and stored at $-80^{\circ} \mathrm{C}$ for later analyses. Survival was analyzed using GraphPad Prism software (GraphPad Software). For xenografts of MEFs, transduced MEFs $\left(2 \times 10^{6}\right.$ cells in $\left.100 \mu \mathrm{l}\right)$ were subcutaneously injected into the flanks of 8-week-old male NSG mice (NOD.Cg-Prkdc $c^{\text {sid }} \mathrm{Il} \mathrm{rg}^{\mathrm{tm} I \mathrm{wjl} /}$ SzJ, purchased from The Jackson Laboratory, stock no. 005557). Two weeks later, tumors were collected and weighed.

Accession numbers. All of the microarray, ChIP-seq, and RNA-seq data sets have been deposited in the NCBI's Gene Expression Omnibus (GEO) database (GEO GSE101854).

Additional methods are described in the Supplemental Information.

Statistics. Unless indicated in the figure legends, an unpaired, 2-tailed Student's $t$ test was used to calculate $P$ values and evaluate the statistical significance of the difference between the indicated samples. A $P$ value of less than 0.05 was considered significant. Fourgroup comparisons were analyzed by 1-factor or 2-factor ANOVA. If the ANOVA was overall significant, a post hoc Student's $t$ test was used for pairwise comparison.

Study approval. All animal procedures were approved by the IACUC of the University of Alabama at Birmingham. 


\section{Author contributions}

$\mathrm{ZY}$ and KS conducted most of the experiments; TB and WL conducted some of the experiments; KG and AKJ conducted bioinformatic analyses; HJ, ZY, KS, and TB designed the experiments; and $\mathrm{HJ}$ wrote the manuscript.

\section{Acknowledgments}

We thank Dirk Eick (Helmholtz Zentrum München, German Research Center for Environmental Health) and Alanna Ruddell (University of Washington) for providing the P493-6 cells, Tim Townes (the University of Alabama at Birmingham) for providing the Raji and Jurkat cells, and the Comprehensive Flow Cytometry Core (CFCC) at the University of Alabama at Birmingham, which is supported by NIH core grants P3O
AR048311 and P30 AI027767. We thank Ying Gai Tusing and Yanfang Zhao for technical assistance with the mice work. This study was supported by American Cancer Society Research Scholar Award 128609-RSG-15-166-01-DMC; start-up funds from the State of Alabama and the University of Virginia; an internal fund from the University of Alabama at Birmingham; and NIH grant R01DK105531. HJ is a recipient of the American Society of Hematology Scholar Award.

Address correspondence to: Hao Jiang, Department of Biochemistry and Molecular Genetics, University of Virginia School of Medicine, P.O. Box 800733, 1340 JPA, Pinn Hall Room 6017, Charlottesville, Virginia 22908, USA. Phone: 434.924.2112; Email: hj8d@virginia.edu.
1. Dang CV. MYC on the path to cancer. Cell. 2012;149(1):22-35.

2. Dalla-Favera R, Bregni M, Erikson J, Patterson D, Gallo RC, Croce CM. Human c-myc onc gene is located on the region of chromosome 8 that is translocated in Burkitt lymphoma cells. Proc Natl Acad Sci U S A. 1982;79(24):7824-7827.

3. Taub R, et al. Translocation of the c-myc gene into the immunoglobulin heavy chain locus in human Burkitt lymphoma and murine plasmacytoma cells. Proc Natl Acad Sci U S A 1982;79(24):7837-7841.

4. Adams JM, et al. The c-myc oncogene driven by immunoglobulin enhancers induces lymphoid malignancy in transgenic mice. Nature. 1985;318(6046):533-538.

5. Rounbehler RJ, et al. Tristetraprolin impairs myc-induced lymphoma and abolishes the malignant state. Cell. 2012;150(3):563-574.

6. Barna M, et al. Suppression of Myc oncogenic activity by ribosomal protein haploinsufficiency. Nature. 2008;456(7224):971-975.

7. Kress TR, Sabò A, Amati B. MYC: connecting selective transcriptional control to global RNA production. Nat Rev Cancer. 2015;15(10):593-607.

8. Lin CY, et al. Transcriptional amplification in tumor cells with elevated c-Myc. Cell. 2012;151(1):56-67.

9. Nie Z, et al. c-Myc is a universal amplifier of expressed genes in lymphocytes and embryonic stem cells. Cell. 2012;151(1):68-79.

10. Sabò A, et al. Selective transcriptional regulation by Myc in cellular growth control and lymphomagenesis. Nature. 2014;511(7510):488-492.

11. Walz S, et al. Activation and repression by oncogenic MYC shape tumour-specific gene expression profiles. Nature. 2014;511(7510):483-487.

12. Guccione E, et al. Myc-binding-site recognition in the human genome is determined by chromatin context. Nat Cell Biol. 2006;8(7):764-770.

13. Soufi A, Donahue G, Zaret KS. Facilitators and impediments of the pluripotency reprogramming factors' initial engagement with the genome. Cell. 2012;151(5):994-1004.

14. Schneider R, Bannister AJ, Myers FA, Thorne AW, Crane-Robinson C, Kouzarides T. Histone H3 lysine 4 methylation patterns in higher eukaryotic genes. Nat Cell Biol. 2004;6(1):73-77.

15. Jiang H, Lu X, Shimada M, Dou Y, Tang Z, Roeder
RG. Regulation of transcription by the MLL2 complex and MLL complex-associated AKAP95. Nat Struct Mol Biol. 2013;20(10):1156-1163.

16. Lauberth SM, et al. H3K4me3 interactions with TAF3 regulate preinitiation complex assembly and selective gene activation. Cell. 2013;152(5):1021-1036.

17. Shilatifard A. The COMPASS family of histone $\mathrm{H} 3 \mathrm{~K} 4$ methylases: mechanisms of regulation in development and disease pathogenesis. Annu Rev Biochem. 2012;81:65-95.

18. Rao RC, Dou Y. Hijacked in cancer: the KMT2 (MLL) family of methyltransferases. Nat Rev Cancer. 2015;15(6):334-346.

19. Okosun J, et al. Integrated genomic analysis identifies recurrent mutations and evolution patterns driving the initiation and progression of follicular lymphoma. Nat Genet. 2014;46(2):176-181.

20. Parsons DW, et al. The genetic landscape of the childhood cancer medulloblastoma. Science. 2011;331(6016):435-439.

21. Pasqualucci L, et al. Analysis of the coding genome of diffuse large B-cell lymphoma. Nat Genet. 2011;43(9):830-837.

22. Rabello Ddo A, de Moura CA, de Andrade RV, Motoyama AB, Silva FP. Altered expression of MLL methyltransferase family genes in breast cancer. Int JOncol. 2013;43(2):653-660.

23. Song $Y$, et al. Identification of genomic alterations in oesophageal squamous cell cancer. Nature. 2014;509(7498):91-95.

24. Lüscher-Firzlaff J, et al. The human trithorax protein hASH2 functions as an oncoprotein. Cancer Res. 2008;68(3):749-758.

25. Kim JY, et al. A role for WDR5 in integrating threonine 11 phosphorylation to lysine 4 methylation on histone $\mathrm{H} 3$ during androgen signaling and in prostate cancer. Mol Cell. 2014;54(4):613-625.

26. Kandoth C, et al. Mutational landscape and significance across 12 major cancer types. Nature. 2013;502(7471):333-339.

27. Jiang H, Shukla A, Wang X, Chen WY, Bernstein BE Roeder RG. Role for Dpy-30 in ES cell-fate specification by regulation of $\mathrm{H} 3 \mathrm{~K} 4$ methylation within bivalent domains. Cell. 2011;144(4):513-525.

28. Yang Z, et al. The DPY30 subunit in SET1/MLL complexes regulates the proliferation and differentiation of hematopoietic progenitor cells. Blood. 2014;124(13):2025-2033.
29. Simboeck E, et al. DPY30 regulates pathways in cellular senescence through ID protein expression. EMBO J. 2013;32(16):2217-2230.

30. Yang Z, Augustin J, Hu J, Jiang H. Physical interactions and functional coordination between the core subunits of Set1/Mll complexes and the reprogramming factors. PLOS ONE. 2015;10(12):e0145336.

31. Yang Z, Shah K, Khodadadi-Jamayran A, Jiang H Dpy30 is critical for maintaining the identity and function of adult hematopoietic stem cells. JExp Med. 2016;213(11):2349-2364.

32. Cerami E, et al. The cBio cancer genomics portal: an open platform for exploring multidimensional cancer genomics data. Cancer Discov. 2012;2(5):401-404

33. Hummel M, et al. A biologic definition of Burkitt's lymphoma from transcriptional and genomic profiling. N Engl J Med. 2006;354(23):2419-2430.

34. Saglio G, et al. 3' c-myc rearrangement in a human leukemic T-cell line. Cancer Res. 1986;46(3):1413-1417.

35. Mu Q, et al.10058-F4, a c-Myc inhibitor, markedly increases valproic acid-induced cell death in Jurkat and CCRF-CEM T-lymphoblastic leukemia cells. Oncol Lett. 2014;8(3):1355-1359.

36. Amati B, Brooks MW, Levy N, Littlewood TD, Evan GI, Land H. Oncogenic activity of the c-Myc protein requires dimerization with Max. Cell. 1993;72(2):233-245.

37. Pajic A, et al. Cell cycle activation by c-myc in a burkitt lymphoma model cell line. Int J Cancer. 2000;87(6):787-793.

38. Cho YW, et al. PTIP associates with MLL3and MLL4-containing histone $\mathrm{H} 3$ lysine 4 methyltransferase complex. J Biol Chem. 2007;282(28):20395-20406.

39. Ullius A, et al. The interaction of MYC with the trithorax protein ASH2L promotes gene transcription by regulating $\mathrm{H} 3 \mathrm{~K} 27$ modification. Nucleic Acids Res. 2014;42(11):6901-6920.

40. Buenrostro JD, Giresi PG, Zaba LC, Chang HY, Greenleaf WJ. Transposition of native chromatin for fast and sensitive epigenomic profiling of open chromatin, DNA-binding proteins and nucleosome position. Nat Methods. 2013;10(12):1213-1218.

41. Vafa O, et al. c-Myc can induce DNA damage, increase reactive oxygen species, and mitigate 
p53 function: a mechanism for oncogene-induced genetic instability. Mol Cell. 2002;9(5):1031-1044.

42. Subramanian A, et al. Gene set enrichment analysis: a knowledge-based approach for interpreting genome-wide expression profiles. Proc Natl Acad Sci USA. 2005;102(43):15545-15550.

43. Dutsch-Wicherek M, Sikora J, Tomaszewska R. The possible biological role of metallothionein in apoptosis. Front Biosci. 2008;13:4029-4038.

44. Shimoda R, et al. Metallothionein is a potential negative regulator of apoptosis. Toxicol Sci. 2003;73(2):294-300.

45. Youle RJ, Strasser A. The BCL-2 protein family: opposing activities that mediate cell death. Nat Rev Mol Cell Biol. 2008;9(1):47-59.

46. Ernst P, Vakoc CR. WRAD: enabler of the SET1-family of H3K 4 methyltransferases. Brief Funct Genomics. 2012;11(3):217-226.

47. Lin DC, et al. Genomic and molecular characterization of esophageal squamous cell carcinoma. Nat Genet. 2014;46(5):467-473.

48. Watanabe Y, et al. Frequent alteration of MLL3 frameshift mutations in microsatellite deficient colorectal cancer. PLoS ONE. 2011;6(8):e23320.

49. Chen C, et al. MLL3 is a haploinsufficient $7 q$ tumor suppressor in acute myeloid leukemia. Cancer Cell. 2014;25(5):652-665.

50. Zhang J, et al. Disruption of KMT2D perturbs ger- minal center B cell development and promotes lymphomagenesis. Nat Med. 2015;21(10):1190-1198.

51. Lee YJ, Han ME, Baek SJ, Kim SY, Oh SO. Roles of DPY30 in the proliferation and motility of gastric cancer cells. PLOS ONE. 2015;10(7):e0131863.

52. Butler JS, et al. Low expression of ASH2L protein correlates with a favorable outcome in acute myeloid leukemia. Leuk Lymphoma. 2017;58(5):1207-1218.

53. Sun Y, et al. WDR5 supports an N-Myc transcriptional complex that drives a protumorigenic gene expression signature in neuroblastoma. Cancer Res. 2015;75(23):5143-5154.

54. Carugo A, et al. In vivo functional platform targeting patient-derived xenografts identifies WDR5-Myc association as a critical determinant of pancreatic cancer. Cell Rep. 2016;16(1):133-147.

55. Shi J, Vakoc CR. The mechanisms behind the therapeutic activity of BET bromodomain inhibition. Mol Cell. 2014;54(5):728-736.

56. Rathert $\mathrm{P}$, et al. Transcriptional plasticity promotes primary and acquired resistance to BET inhibition. Nature. 2015;525(7570):543-547.

57. Fong CY, et al. BET inhibitor resistance emerges from leukaemia stem cells. Nature. 2015;525(7570):538-542.

58. Soufi A, Garcia MF, Jaroszewicz A, Osman N, Pellegrini M, Zaret KS. Pioneer transcription factors target partial DNA motifs on nucleosomes to initiate reprogramming. Cell. 2015;161(3):555-568.

59. Dou Y, et al. Regulation of MLL1 H3K4 methyltransferase activity by its core components. Nat Struct Mol Biol. 2006;13(8):713-719.

60. Thomas LR, et al. Interaction with WDR5 promotes target gene recognition and tumorigenesis by MYC. Mol Cell. 2015;58(3):440-452.

61. Richart L, et al. BPTF is required for c-MYC transcriptional activity and in vivo tumorigenesis. Nat Commun. 2016;7:10153.

62. Ruthenburg AJ, Allis CD, Wysocka J. Methylation of lysine 4 on histone H3: intricacy of writing and reading a single epigenetic mark. Mol Cell. 2007;25(1):15-30.

63. Tremblay V, et al. Molecular basis for DPY-30 association to COMPASS-like and NURF complexes. Structure. 2014;22(12):1821-1830.

64. Kelly PN, Grabow S, Delbridge AR, Strasser A, Adams JM. Endogenous Bcl-xL is essential for Myc-driven lymphomagenesis in mice. Blood. 2011;118(24):6380-6386.

65. Kelly PN, Puthalakath H, Adams JM, Strasser A. Endogenous bcl-2 is not required for the development of Emu-myc-induced B-cell lymphoma. Blood. 2007;109(11):4907-4913.

66. Dawson MA, Kouzarides T. Cancer epigenetics: from mechanism to therapy. Cell. 2012;150(1):12-27. 